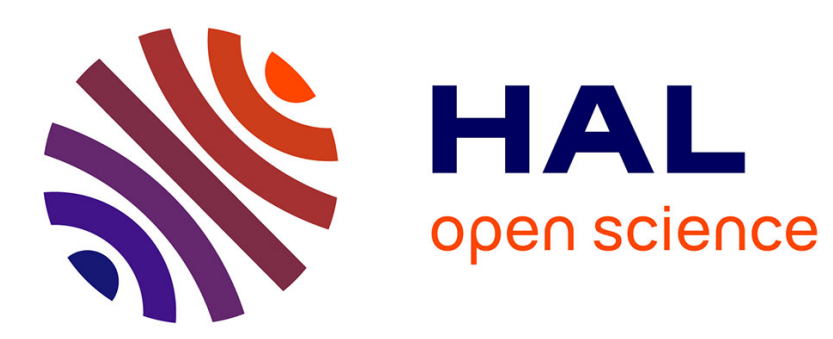

\title{
Robust output-feedback control for uncertain linear sampled-data systems: A 2D impulsive system approach
}

Héctor Ríos, Laurentiu Hetel, Denis Efimov

\section{To cite this version:}

Héctor Ríos, Laurentiu Hetel, Denis Efimov. Robust output-feedback control for uncertain linear sampled-data systems: A 2D impulsive system approach. Nonlinear Analysis: Hybrid Systems, 2019, 32, pp.177-201. 10.1016/j.nahs.2018.11.005 . hal-01954885

\section{HAL Id: hal-01954885 \\ https://hal.science/hal-01954885}

Submitted on 7 Feb 2019

HAL is a multi-disciplinary open access archive for the deposit and dissemination of scientific research documents, whether they are published or not. The documents may come from teaching and research institutions in France or abroad, or from public or private research centers.
L'archive ouverte pluridisciplinaire $\mathbf{H A L}$, est destinée au dépôt et à la diffusion de documents scientifiques de niveau recherche, publiés ou non, émanant des établissements d'enseignement et de recherche français ou étrangers, des laboratoires publics ou privés. 


\title{
Robust Output-Feedback Control for Uncertain Linear Sampled-Data

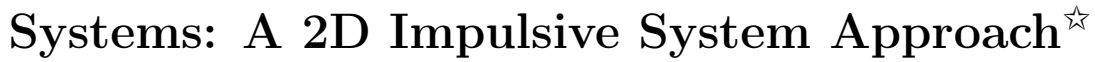

\author{
Héctor Ríos ${ }^{\mathrm{a}, *}$, Laurentiu Hetel ${ }^{\mathrm{b}}$, and Denis Efimov ${ }^{\mathrm{b}, \mathrm{c}}$ \\ ${ }^{a}$ CONACYT-TECNM/Instituto Tecnológico de la Laguna, División de Estudios de Posgrado e Investigación, Blvd. Revolución y \\ Cuauhtémoc S/N, C.P. 27000, Torreón, Coahuila, México. \\ ${ }^{b}$ CRIStAL (UMR-CNRS 9189), Ecole Centrale de Lille, BP 48, Cité Scientifique, 59651 Villeneuve-d'Ascq, France. \\ ${ }^{c}$ Non-A team @ Inria, Parc Scientifique de la Haute Borne, 40 avenue Halley, 59650 Villeneuve d'Ascq, France; and Department of \\ Control Systems and Informatics, Saint Petersburg State University of Information Technologies Mechanics and Optics (ITMO), \\ Kronverkskiy av. 49, Saint Petersburg, 197101, Russia.
}

\begin{abstract}
This paper deals with the sampled-data control problem based on state estimation for uncertain linear sampled-data systems. It is possible to show that the sampled-data control problem based on state estimation may be related with the conditions for the exponential stability of impulsive systems. Thus, a vector Lyapunov function-based approach, derived by means of a $2 D$ time domain equivalence, is used for obtaining stability conditions of an impulsive system, and then, a solution to the observer-based control design problem is derived and expressed in terms of LMIs. Some examples illustrate the feasibility of the proposed approach.
\end{abstract}

Keywords: Observer-based Control, Sampled-Data, Impulsive systems.

\section{Introduction}

In the last decades, an enormous interest has appeared in the design of controllers and observers for continuous and/or discrete dynamical systems with communication constraints. This interest has its motivations in systems with sampled-data control, quantization and more generally, in networked control systems. However, all the communications constraints, i.e. delays, sampling intervals, quantization, packet dropouts, and so on (for details, see [16]); imply additional difficulties in the analysis and design compared to the classical control systems. Regarding the observer design problem, one of the main issues is the scheduling: only a subset of sensors is allowed to send their data to the observer at the transmission instants. The sporadic and partial availability of system measurements requires the development of appropriate observer designs. Moreover, for controller design, it would be unreasonable to assume that all states are measurable. Therefore an observer-based control approach is needed.

In this paper the observer-based control problem will be in the focus for sampled-data systems. Several methods have been developed to study sampled-data systems, e.g. the Input/Output stability approach [14], the discretetime approach [13], but two approaches stand out: the input delay approach, where the system is modeled as a continuous system with a delay in the control input (see, e.g. $[10,11])$, and the impulsive system approach, where the sampled-data system is treated as an impulsive system (see, e.g. [3, 4, 5, 29]).

The input delay approach has been applied in [12] to design a sampled-data output-feedback $H_{\infty}$ control for linear systems while the impulsive system approach was applied in [17] to sampled-data stabilization of linear uncertain systems in the case of constant sampling based on piecewise linear in time Lyapunov function. The case of variable sampling based on a discontinuous Lyapunov function method was introduced by [23]. Also based on discontinuous Lyapunov functions, in [3] stability and stabilization conditions for periodic and aperiodic sampled-data systems are introduced.

\footnotetext{
${ }^{\sqrt{4}} \mathrm{H}$. Ríos gratefully acknowledges the financial support from CONACyT 270504. This work was supported in part by Conseil Region Nord-Pas de Calais (ESTIREZ), the Government of Russian Federation (Grant 074-U01), the Ministry of Education and Science of Russian Federation (Project 14.Z50.31.0031), and ANR ROCC-SYS (ANR-14-CE27-0008).

* Corresponding author

Email addresses: hriosb@correo.itlalaguna.edu.mx (Héctor Ríos), laurentiu.hetel@ec-lille.fr (Laurentiu Hetel), denis.efimov@inria.fr (and Denis Efimov)
} 
In the context of observer design, one approach is based on continuous and discrete design. In [7], such an approach is used to design a continuous-discrete version of the high-gain observer for nonlinear systems. In [18] a continuous-discrete observer is proposed for linear and triangular Lipschitz systems based on a sampled-data nonlinear observer that is designed using a continuous-time approach together with an inter-sample output predictor. Applying a small gain approach, in [1] an observer design is proposed for certain classes of nonlinear systems with sampled and delayed measurements. A Luenberger-like observer is proposed by [8] for a class of continuous-time dynamical systems with non-uniformly sampled measurements. In [22], continuous-time systems with sampled uncertain output are considered and the state estimation problem is solved by means of continuous-discrete interval observers that are asymptotically stable in the absence of disturbances. In [21], based on the notion of cooperative systems, a design for continuous-discrete observers is proposed for continuous nonlinear time-varying systems with discrete-time measurements. Using the hybrid system approach, in [6] an observer-protocol pair is designed to estimate the states of a linear system under communication constraints induced by the network. In the same vein, in [9] an observer with jumps triggered by incoming measurements is proposed to deal with the state estimation problem for linear time-invariant systems for which measurements of the output are available sporadically. Adopting a switched observer structure, in [2] decentralized observer-based output-feedback controllers are proposed for linear systems connected via a shared communication network.

In this paper a vector Lyapunov function-based approach [19], derived by means of a $2 D$ time domain equivalence (see, e.g. [28] and [31]), for stability of impulsive systems is used for designing a robust output-feedback control for linear sampled-data systems. Such an approach, proposed in [26] and [27], provides a stability analysis based on linear matrix inequalities (LMIs) for linear impulsive dynamical systems. Then, it is possible to show that the sampled-data control problem based on state estimation may turn into one of finding conditions for the exponential stability of impulsive systems. Thus, the proposed vector Lyapunov function approach is applied for obtaining stability conditions of the impulsive system, and then, a solution to the robust output-feedback control design problem is obtained and expressed in terms of LMIs. To the best of our knowledge, the output-feedback control design for uncertain sampled-data system is open in the literature and there exist very few works dealing with such a problem. Moreover, it is worth highlighting that a direct application of the methods given in the literature, e.g. those ones from [3], [4], or in [15], do not provide a constructive method to solve the robust output-feedback control design problem for uncertain linear sampled-data systems.

The outline of this work is as follows. A motivating problem is given in Section 2. Some stability results for impulsive systems are given in Section 3. The main result is described in Section 4. Some simulation results are depicted in Section 5 while some concluding remarks are discussed in Section 6. The corresponding proofs for the main results are postponed to the Appendix.

\section{Motivation}

Let us consider the following uncertain sampled-data system

$$
\begin{aligned}
\dot{x}(t) & =A x(t)+B u(t)+f(x(t)), x(0)=x_{0}, \\
y(t) & =C x\left(t_{i}\right), \forall t \in\left[t_{i}, t_{i+1}\right), \\
u(t) & =K \hat{x}\left(t_{i}\right), \forall t \in\left[t_{i}, t_{i+1}\right),
\end{aligned}
$$

where $x, x_{0} \in \mathbb{R}^{n}$ are the state vector and the initial condition, respectively, $u \in \mathbb{R}^{m}$ is the sampled control vector, and $y \in \mathbb{R}^{p}$ is the sampled output vector at each time $t_{i}$ for all $i \in \mathbb{N}$, and $\hat{x} \in \mathbb{R}^{n}$ represents an estimation of the system state $x$. The function $f: \mathbb{R}^{n} \rightarrow \mathbb{R}^{n}$ represents all the parameter uncertainties of the system satisfying $|f(x)|^{2} \leq f_{0}|x|^{2}$, i.e. the function $f$ is Lipschitz. The constant matrices $A, B$, and $C$ have corresponding dimensions while $K$ is a design control matrix.

The sampling instants $t_{i}$ are monotonously increasing, such that $\lim _{i \rightarrow \infty} t_{i}=+\infty$, and $T_{i}:=t_{i+1}-t_{i} \in$ $\left[T_{\min }, T_{\max }\right]$, where $T_{\min }>0$ and $T_{\max }>0$ are the minimum and maximum sampling intervals, respectively; and $t_{0}=0$. The control $u$ is designed by means of the following sampled-data state observer

$$
\dot{\hat{x}}(t)=A \hat{x}(t)+B u(t)+L\left(y(t)-C \hat{x}\left(t_{i}\right)\right), \forall t \in\left[t_{i}, t_{i+1}\right), \hat{x}(0)=\hat{x}_{0},
$$

where $\hat{x}, \hat{x}_{0} \in \mathbb{R}^{n}$ are the estimated state vector and its initial condition, and $L \in \mathbb{R}^{n \times p}$ is a design observer matrix. Define the state estimation error $e(t):=x(t)-\hat{x}(t)$. Then, the closed-loop and state estimation error dynamics are 
given as follows

$$
\begin{aligned}
& \dot{x}(t)=A x(t)+B K x\left(t_{i}\right)-B K e\left(t_{i}\right)+f(x(t)), \forall t \in\left[t_{i}, t_{i+1}\right), \\
& \dot{e}(t)=A e(t)-L C e\left(t_{i}\right)+f(x(t)), \forall t \in\left[t_{i}, t_{i+1}\right) .
\end{aligned}
$$

Let us define the extended state vector $\xi(t):=\left(x^{T}(t) e^{T}(t) x^{T}\left(t_{i}\right) e^{T}\left(t_{i}\right)\right)^{T} \in \mathbb{R}^{4 n}$ and the timer variable $\tau \in \mathbb{R}_{\geq 0}$. Then, the above dynamics may be written as follows

$$
\begin{aligned}
\frac{d}{d t}\left(\begin{array}{c}
\xi \\
\tau
\end{array}\right) & =\left(\begin{array}{c}
A_{\xi} \xi+D_{\xi} f \\
1
\end{array}\right), \forall \tau \in\left[0, T_{i}\right), i=0,1,2, \ldots,(\xi(0), \tau(0))=\left(\xi_{0}, \tau_{0}\right), \\
\left(\begin{array}{c}
\xi^{+} \\
\tau^{+}
\end{array}\right) & =\left(\begin{array}{c}
I_{\xi} \xi \\
0
\end{array}\right), \forall \tau=T_{i}, i=0,1,2, \ldots
\end{aligned}
$$

where $(\xi, \tau),\left(\xi_{0}, \tau_{0}\right) \in \mathbb{R}^{4 n} \times\left[0, T_{i}\right]$ represents the current state vector and its initial condition, $\left(\xi^{+}, \tau^{+}\right) \in \mathbb{R}^{4 n} \times T_{i}$ represents the reset state vector, $T_{i} \in\left[T_{\min }, T_{\max }\right]$ is the sampling interval given for $i=0,1,2, \ldots$, and $f \in \mathbb{R}^{n}$ denotes the uncertainty. The corresponding matrices have the following structure

$$
A_{\xi}=\left(\begin{array}{cccc}
A & \mathbf{0} & B K & -B K \\
\mathbf{0} & A & \mathbf{0} & -L C \\
\mathbf{0} & \mathbf{0} & \mathbf{0} & \mathbf{0} \\
\mathbf{0} & \mathbf{0} & \mathbf{0} & \mathbf{0}
\end{array}\right), D_{\xi}=\left(\begin{array}{c}
I_{n} \\
I_{n} \\
\mathbf{0} \\
\mathbf{0}
\end{array}\right), I_{\xi}=\left(\begin{array}{cccc}
I_{n} & \mathbf{0} & \mathbf{0} & \mathbf{0} \\
\mathbf{0} & I_{n} & \mathbf{0} & \mathbf{0} \\
I_{n} & \mathbf{0} & \mathbf{0} & \mathbf{0} \\
\mathbf{0} & I_{n} & \mathbf{0} & \mathbf{0}
\end{array}\right)
$$

Note that in absence of uncertainties, i.e. $f=0$, the set $\left\{(\xi, \tau) \mid \xi=0, \tau \in\left[0, T_{i}\right]\right\}$ is an equilibrium set of (5). The dynamics (5)-(6) describes periodic/aperiodic time-triggered jumps, when $\tau=T_{i}$, governed by the map given by (6), while between the jumps the system behaves according to (5). Note also that, due to the linearity of the system and the facts that $f$ is Lipschitz and $T_{i} \in\left[T_{\min }, T_{\max }\right]$, the existence of a unique forward solution is ensured.

Then, the sampled-data control problem based on state estimation, i.e. find the control gain matrix $K$ and the observer gain matrix $L$, may turn into one of finding conditions for the stability of the impulsive system described by (5)-(6), under arbitrary variations of the sampling intervals.

In the following sections such conditions for the stability of the impulsive system are derived by means of a $2 D$ time domain equivalence and a vector Lyapunov function approach. Afterwards, these conditions will be applied to solve the sampled-data control problem based on state estimation. All the proofs are given in the Appendix.

\section{Stability Analysis for Impulsive Systems}

The stability analysis relies on the embedding of system (5)-(6) into a $2 D$ time domain. Indeed, the entire state trajectory $(\xi, \tau)$ can be viewed as a sequence of the diagonal dynamics ${ }^{1}$ of the following $2 D$ system:

$$
\begin{aligned}
& \frac{d}{d t}\left(\begin{array}{c}
\xi_{k}^{t} \\
\tau_{k}^{t}
\end{array}\right)=\left(\begin{array}{c}
A_{\xi} \xi_{k}^{t}+D_{\xi} f \\
1
\end{array}\right), \forall \tau_{k}^{t} \in\left[0, T_{i}\right), \forall i=k=0,1,2, \ldots,(\xi(0,0), \tau(0,0))=\left(\xi_{0}^{0}, \tau_{0}^{0}\right) \\
& \left(\begin{array}{c}
\xi_{k+1}^{t_{i+1}} \\
\tau_{k+1}^{t_{i+1}}
\end{array}\right)=\left(\begin{array}{c}
I_{\xi} \xi_{k}^{t_{i+1}} \\
0
\end{array}\right), \forall \tau_{k}^{t}=T_{i}, \forall i=k=0,1,2, \ldots
\end{aligned}
$$

where $\left(\xi_{k}^{t}, \tau_{k}^{t}\right):=(\xi(t, k), \tau(t, k)),\left(\xi_{0}^{0}, \tau_{0}^{0}\right) \in \mathbb{R}^{4 n} \times\left[0, T_{i}\right]$ is the current state vector and its initial condition, $\left(\xi_{k+1}^{t_{i+1}}, \tau_{k+1}^{t_{i+1}}\right):=\left(\xi\left(t_{i+1}, k+1\right), \tau\left(t_{i+1}, k+1\right)\right) \in \mathbb{R}^{4 n} \times T_{i}$ represents the reset state vector, while $\left(\xi_{k}^{t_{i+1}}, \tau_{k}^{t_{i+1}}\right):=$ $\left(\xi^{T}\left(t_{i+1}, k\right), \tau\left(t_{i+1}, k\right)\right)^{T} \in \mathbb{R}^{4 n} \times T_{i}$ denotes the value of $(\xi, \tau)$ just before the jump $k+1$. Taking into account that $f$ is Lipschitz and $T_{i} \in\left[T_{\min }, T_{\max }\right]$, the solutions of (7)-(8) for the diagonal dynamics, i.e. for all $i=k$, correspond to the solutions of the system (5)-(6). Note that the discrete time $k$ depicts the number of impulses in the system.

In the present section some definitions and results for the stability of impulsive systems, in the framework of $2 D$ systems, are introduced (see [26] and [27]).

\footnotetext{
${ }^{1}$ The diagonal dynamics make reference only to those dynamics given by (7)-(8) corresponding to $i=k$, for all $i, k \in \mathbb{N}$ and for all $t \in \mathbb{R}_{\geq 0}$.
} 
Let $|q|$ denote the Euclidean norm of a vector $q$. The following stability definition is introduced:

Definition 1. [26]. A 2D system described by (7)-(8), is said to be exponentially diagonal $\xi_{k}^{t}$-stable (ED $\xi_{k}^{t}$-S) if there exist positive constants $\kappa_{1}, \kappa_{2}, \kappa_{3}$, and $c$ such that $0<\kappa_{1}<1$ and

$$
\begin{gathered}
\left|\xi_{k+1}^{t_{i+1}}\right|^{2} \leq c \kappa_{1}^{k+1}\left|\xi_{0}^{0}\right|^{2}, \quad \forall \tau_{k}^{t}=T_{i}, \\
\left|\xi_{k}^{t}\right|^{2} \leq \kappa_{2}\left|\xi_{k}^{t_{i}}\right|^{2}, \quad \forall \tau_{k}^{t} \in\left[0, T_{i}\right], \\
\left|\tau_{k}^{t}\right| \leq \kappa_{3},
\end{gathered}
$$

for all $i=k \in \mathbb{N}$.

Note that condition (11) holds by definition, i.e. $\left|\tau_{k}^{t}\right| \leq \kappa_{3}$, with $\kappa_{3}=T_{\max }$. Denote $z_{k}^{t}:=\left(\left(\xi_{k}^{t}\right)^{T}, \tau_{k}^{t}\right)^{T}$. In order to give the stability conditions a vector Lyapunov approach is used, i.e.

$$
V\left(z_{k}^{t}, z_{k+1}^{t_{i+1}}\right)=\left(\begin{array}{c}
V_{1}\left(z_{k}^{t}\right) \\
V_{2}\left(z_{k+1}^{t_{i+1}}\right)
\end{array}\right)
$$

where $V_{1}(\cdot)>0, V_{2}(\cdot)>0$, for all $z_{k}^{t}$ and $z_{k+1}^{t_{i+1}}$, and $V_{1}(0)=0, V_{2}(0)=0$. Now, let us introduce the following definition.

Definition 2. The divergence operator of a function $V$ along the trajectories of system (7)-(8) is defined for all $t \in\left[t_{i}, t_{i+1}\right)$ as follows

$$
\operatorname{div} V\left(z_{k}^{t}, z_{k+1}^{t_{i+1}}\right)=\frac{d V_{1}\left(z_{k}^{t}\right)}{d t}+V_{2}\left(z_{k+1}^{t_{i+1}}\right)-V_{2}\left(z_{k}^{t_{i+1}}\right)
$$

Note that $V_{1}$ is differentiable with respect to continuous time $t$ while the difference in $V_{2}$ is calculated in discrete time $k$. Thus, the following theorem is introduced.

Theorem 1. [26]. Assume that there exist positive constants $\varepsilon, c_{1}, c_{2}, c_{3}, c_{4}$ and $c_{5}$ such that the vector Lyapunov function $V\left(z_{k}^{t}, z_{k+1}^{t_{i+1}}\right)$ and its divergence along the trajectories of the system (7)-(8) satisfy, for all $\tau_{k}^{t} \in\left[0, T_{i}\right]$, $i=k \in \mathbb{N}$, the following inequalities:

$$
\begin{gathered}
c_{1}\left|\xi_{k}^{t}\right|^{2} \leq V_{1}\left(z_{k}^{t}\right) \leq c_{2}\left|\xi_{k}^{t}\right|^{2}, \\
c_{3}\left|\xi_{k}^{p}\right|^{2} \leq V_{2}\left(z_{k}^{p}\right) \leq c_{4}\left|\xi_{k}^{p}\right|^{2}, \forall p=t_{i}, t_{i+1} \\
\operatorname{div} V \leq-c_{5}\left(\left|\xi_{k}^{t}\right|^{2}+\left|\xi_{k}^{t_{i+1}}\right|^{2}\right), \\
c_{2}\left(c_{4}-c_{5}\right) \leq c_{1} c_{5} \vee T_{i} \leq \frac{c_{2}}{c_{5}} \alpha, \\
\frac{c_{2}}{c_{5}} \gamma \leq T_{i},
\end{gathered}
$$

where $\gamma=-\ln \left[\frac{c_{3}(1-\varepsilon)}{c_{5}+c_{3}(1-\varepsilon)}\right]$ and $\alpha=-\ln \left[\frac{c_{2}\left(c_{4}-c_{5}\right)-c_{1} c_{5}}{c_{2}\left(c_{4}-c_{5}\right)}\right]$ for all $c_{2}\left(c_{4}-c_{5}\right)>c_{1} c_{5}$. Then, the $2 D$ system $(7)-(8)$ is $\mathrm{ED} \xi_{k}^{t}-\mathrm{S}$ for any sequence $\left\{T_{i}\right\}_{i \in \mathbb{N}}$ such that $T_{i} \in\left[\frac{c_{2}}{c_{5}} \gamma, \frac{c_{2}}{c_{5}} \alpha\right]$.

The statement given by Theorem 1 relies on a vector Lyapunov function approach in contrast to the results given in [15] (similarly in [24]), where asymptotic stability is obtained by means of a single Lyapunov function that needs to have a negative semi-definite derivative. Alternatively, our divergence operator, and not each term, needs to satisfy inequality (15).

Remark 1. The constructive application of Theorem 1 is illustrated by Algorithm 1 which provides some notions of minimum and maximum or ranged dwell-time depending on the structure of the system dynamics. In particular, the first and third cases for exponential diagonal stability (pseudo-code lines: 5 and 13, Algorithm 1) give conditions for minimum dwell-time while the second case (pseudo-code lines: 7, Algorithm 1) provides conditions for maximum or ranged dwell-time. 


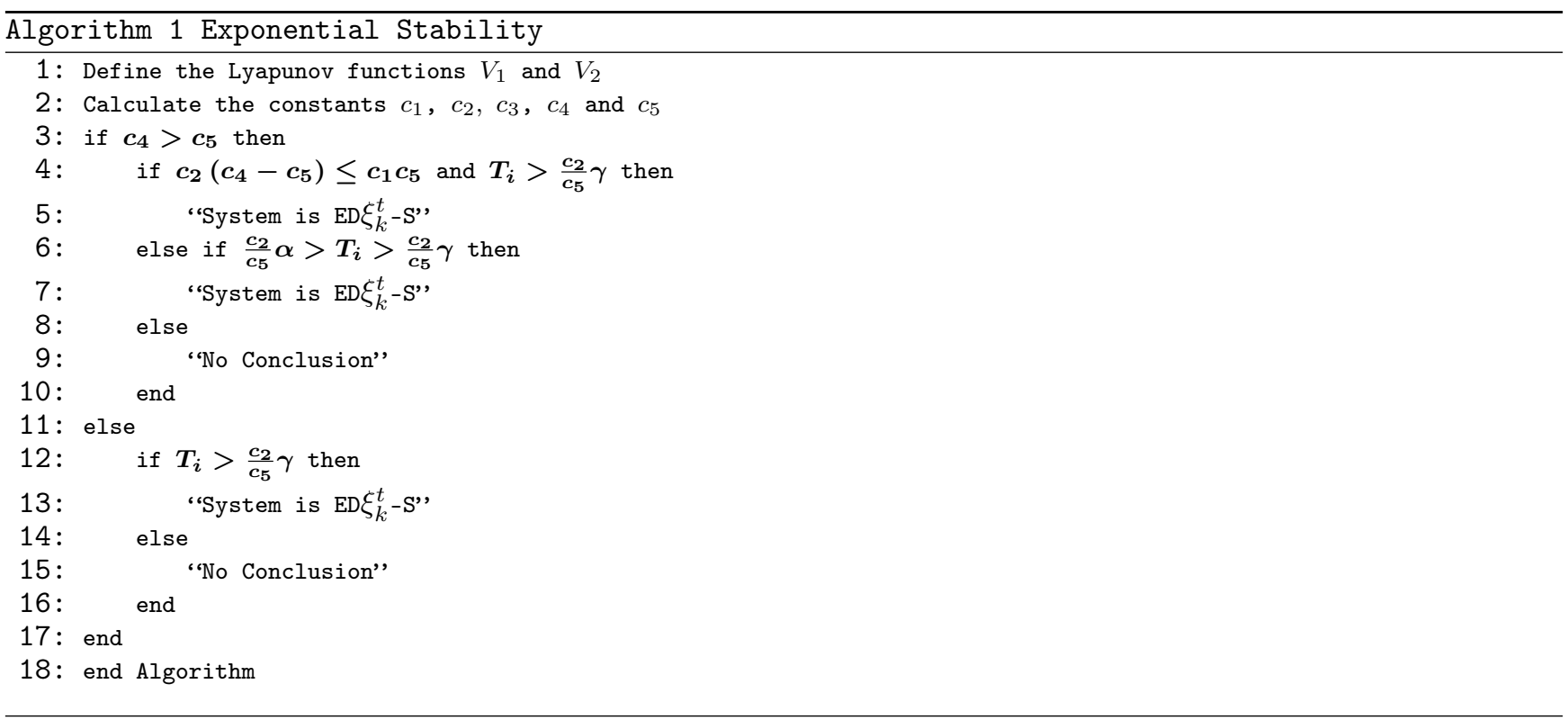

\subsection{Exponential Diagonal $\xi_{k}^{t}$-Stability: Quadratic Lyapunov Functions}

Consider that $V_{1}$ and $V_{2}$ take the following quadratic structure

$$
V\left(z_{k}^{t}, z_{k+1}^{t_{i+1}}\right)=\left(\begin{array}{c}
\left(\xi_{k}^{t}\right)^{T} P_{1}\left(\tau_{k}^{t}\right) \xi_{k}^{t} \\
\left(\xi_{k+1}^{t_{i+1}}\right)^{T} P_{2}\left(\tau_{k+1}^{t_{i+1}}\right) \xi_{k+1}^{t_{i+1}}
\end{array}\right)
$$

where $P_{1} \in \mathbb{R}^{4 n \times 4 n}$ is continuously differentiable with respect to $t$, symmetric, bounded, and positive definite matrix for all $\tau_{k}^{t} \in\left[0, T_{i}\right], i=k \in \mathbb{N}$, while $P_{2} \in \mathbb{R}^{4 n \times 4 n}$ is a symmetric and positive definite matrix, i.e.

$$
\begin{gathered}
0<c_{1} I \leq P_{1}\left(\tau_{k}^{t}\right) \leq c_{2} I, \forall \tau_{k}^{t} \in\left[0, T_{i}\right], \\
0<c_{3} I \leq P_{2}\left(\tau_{k}^{p}\right) \leq c_{4} I, p=t_{i}, t_{i+1} .
\end{gathered}
$$

Thus, based on the previous choice for $V_{1}$ and $V_{2}$, if Theorem 1 is applied to the ideal and uncertain impulsive system (5)-(6), then the following results are obtained.

Corollary 1. Consider the vector Lyapunov function $V\left(z_{k}^{t}, z_{k+1}^{t_{i+1}}\right)$ in (18). Assume that there exist matrices $P_{1}\left(\tau_{k}^{t}\right)=P_{1}^{T}\left(\tau_{k}^{t}\right)>0$, continuously differentiable on $t$ and bounded for all $\tau_{k}^{t} \in\left[0, T_{i}\right], i=k \in \mathbb{N}, P_{2}(0)=P_{2}^{T}(0)>0$ and $P_{2}\left(T_{i}\right)=P_{2}^{T}\left(T_{i}\right)>0$ satisfying (19)-(20), $\Lambda=\Lambda^{T}>0$ and a constant $c_{5}>0$, such that the following matrix inequality

$$
\left(\begin{array}{ccc}
P_{1}\left(\tau_{k}^{t}\right) A_{\xi}+A_{\xi}^{T} P_{1}\left(\tau_{k}^{t}\right)+\frac{d P_{1}\left(\tau_{k}^{t}\right)}{d t}+2 f_{0} \Lambda+c_{5} I_{4 n} & \mathbf{0} & P_{1}\left(\tau_{k}^{t}\right) \\
\star & I_{\xi}^{T} P_{2}(0) I_{\xi}-P_{2}\left(T_{i}\right)+c_{5} I_{4 n} & \mathbf{0} \\
\star & \star & -\Lambda
\end{array}\right) \leq 0,
$$

holds for all $\tau_{k}^{t} \in\left[0, T_{i}\right], i=k \in \mathbb{N},|f|^{2} \leq f_{0}|x|^{2}$, and constraints (16)-(17) are satisfied with $c_{1}, c_{2}, c_{3}, c_{4}$ and $c_{5}$. Then the system (7)-(8) is $\mathrm{ED} \xi_{k}^{t}-\mathrm{S}$ for any sequence $\left\{T_{i}\right\}_{i \in \mathbb{N}}$ such that $T_{i} \in\left[\frac{c_{2}}{c_{5}} \gamma, \frac{c_{2}}{c_{5}} \alpha\right]$.

Now, the following result is established for the ideal impulsive system, i.e. $f=0$.

Corollary 2. Consider the vector Lyapunov function $V\left(z_{k}^{t}, z_{k+1}^{t_{i+1}}\right)$ in (18). Assume that there exist matrices $P_{1}\left(\tau_{k}^{t}\right)=P_{1}^{T}\left(\tau_{k}^{t}\right)>0$, continuously differentiable on $t$ and bounded for all $\tau_{k}^{t} \in\left[0, T_{i}\right], i=k \in \mathbb{N}, P_{2}(0)=P_{2}^{T}(0)>0$ and $P_{2}\left(T_{i}\right)=P_{2}^{T}\left(T_{i}\right)>0$ satisfying (19)-(20), and a constant $c_{5}>0$, such that the following matrix inequality

$$
\left(\begin{array}{cc}
P_{1}\left(\tau_{k}^{t}\right) A_{\xi}+A_{\xi}^{T} P_{1}\left(\tau_{k}^{t}\right)+\frac{d P_{1}\left(\tau_{k}^{t}\right)}{d t}+c_{5} I_{4 n} & \mathbf{0} \\
\mathbf{0} & I_{\xi}^{T} P_{2}(0) I_{\xi}-P_{2}\left(T_{i}\right)+c_{5} I_{4 n}
\end{array}\right) \leq 0,
$$


holds for all $\tau_{k}^{t} \in\left[0, T_{i}\right]$, for all $i=k \in \mathbb{N}$, and constraints (16)-(17) are satisfied with $c_{1}, c_{2}, c_{3}, c_{4}$ and $c_{5}$. Then the system (7)-(8), with $f=0$, is $\mathrm{ED} \xi_{k}^{t}-\mathrm{S}$ for any sequence $\left\{T_{i}\right\}_{i \in \mathbb{N}}$ such that $T_{i} \in\left[\frac{c_{2}}{c_{5}} \gamma, \frac{c_{2}}{c_{5}} \alpha\right]$.

For the particular case of linear time-invariant systems, our method can be seen as a generalization of the result in [3]. In fact, taking $P_{1}\left(\tau_{k}^{t}\right)$ and $P_{2}\left(\tau_{k}^{t}\right)$ in the same form for the statements given by Corollary 2 , one leads to the conditions given by Theorem 2.2 (ranged dwell-time) and 2.3 (minimum dwell-time) in [3].

It is also worth mentioning that the results given by Theorem 1 and Corollary 2 are consistent with the ones in Proposition 3.24 (Persistent Flowing) and Proposition 3.27 (Persistent Jumping) in [15], respectively.

Note that Corollaries 1 and 2 are able to deal with linear impulsive systems where matrix $A_{\xi}$ is not Hurwitz, and/or $I_{\xi}$ is anti-Schur, respectively. In this sense, Corollaries 1 and 2 provide general results to deal with the stability of linear impulsive systems.

In the following, a couple of examples, taken from [5], are presented in the framework of stability, not in the one of output-feedback control design, to illustrate the potential of the proposed method.

Example 1. Let us consider a system as in (7)-(8) with the following matrices

$$
A_{\xi}=\left(\begin{array}{cc}
1 & 3 \\
-1 & 2
\end{array}\right), \quad I_{\xi}=\left(\begin{array}{cc}
0.5 & 0 \\
0 & 0.5
\end{array}\right)
$$

Note that the continuous dynamics is unstable while the discrete one is stable, i.e. $A_{\xi}$ is not Hurwitz and $I_{\xi}$ is Schur. Corollary 2 with

$$
P_{1}\left(\tau_{k}^{t}\right)=\frac{\tau_{k}^{t} P_{11}+\left(T_{i}-\tau_{k}^{t}\right) P_{12}}{T_{i}}, P_{2}\left(\tau_{k}^{t}\right)=P_{21}+\tau_{k}^{t} P_{22}
$$

and a bisection algorithm on $\tau_{k}^{t} \in\left[0, T_{i}\right]$ can be checked by solving LMIs given by (22). The following results are obtained:

$$
P_{11}=\left(\begin{array}{ll}
1.3914 & 0.0139 \\
0.0139 & 1.3597
\end{array}\right), P_{12}=10^{4}\left(\begin{array}{cc}
1.2638 & -0.6319 \\
-0.6319 & 3.7917
\end{array}\right), P_{21}=\left(\begin{array}{cc}
1.0007 & 0 \\
0 & 1.0007
\end{array}\right), P_{22}=\left(\begin{array}{cc}
0.9336 & 0 \\
0 & 0.9336
\end{array}\right),
$$

with $c_{1}=1.3545, c_{2}=3.9410 \times 10^{4}, c_{3}=1.0007, c_{4}=1.3118$ and $c_{5}=0.5000$. Therefore, according to Corollary 2 , the impulsive system is $\mathrm{ED} \xi_{k}^{t}$-S for all $0.3333>T_{i}>0$. It is easy to check that $c_{4}>c_{5}$ and $c_{2}\left(c_{4}-c_{5}\right)>c_{1} c_{5}$ hold and then, according to Algorithm 1, the second case for $\mathrm{ED} \xi_{k}^{t}$-S is obtained. When, as in [5], the analysis is restricted to single quadratic Lyapunov functions linear in $\tau_{k}^{t}$, i.e. $P_{1}\left(\tau_{k}^{t}\right)=P_{2}\left(\tau_{k}^{t}\right)$, it is possible to show stability for $0.2400>T_{i}>0$.

Example 2. Let us consider a system as in (7)-(8) with the following matrices

$$
A_{\xi}=\left(\begin{array}{cc}
-1 & 0 \\
1 & -2
\end{array}\right), \quad I_{\xi}=\left(\begin{array}{cc}
2 & 1 \\
1 & 3
\end{array}\right)
$$

For this example the continuous dynamics is stable while the discrete one is unstable, i.e. $A_{\xi}$ is Hurwitz and $I_{\xi}$ is anti-Schur. Corollary 2 with

$$
P_{1}\left(\tau_{k}^{t}\right)=\frac{\tau_{k}^{t} P_{11}+\left(T_{i}-\tau_{k}^{t}\right) P_{12}}{T_{i}}, P_{2}\left(\tau_{k}^{t}\right)=P_{21}+\tau_{k}^{t} P_{22}
$$

and a bisection algorithm on $\tau_{k}^{t} \in\left[0, T_{i}\right]$ can be checked by solving LMIs given by (22). The following results are obtained:

$$
P_{11}=\left(\begin{array}{ll}
3.3096 & 0.4527 \\
0.4527 & 1.4411
\end{array}\right), P_{12}=\left(\begin{array}{ll}
0.6648 & 0.0963 \\
0.0963 & 0.3941
\end{array}\right), P_{21}=\left(\begin{array}{cc}
0.1123 & 0 \\
0 & 0.1123
\end{array}\right), P_{22}=\left(\begin{array}{ll}
0.0050 & 0.0006 \\
0.0006 & 0.0056
\end{array}\right),
$$

with $c_{1}=1.3372, c_{2}=4.1071, c_{3}=0.1123, c_{4}=6.05$ and $c_{5}=4.58$. Therefore, according to Corollary 2 , the impulsive system is $\mathrm{ED} \xi_{k}^{t}-\mathrm{S}$ for all $T_{i}>3.3254$. It is easy to check that $c_{4}>c_{5}$ and $c_{2}\left(c_{4}-c_{5}\right) \leq c_{1} c_{5}$ hold and then, according to Algorithm 1, the first case for $\mathrm{ED} \xi_{k}^{t}-\mathrm{S}$ is obtained. When, as in [5], the analysis is restricted to single quadratic Lyapunov functions linear in $\tau_{k}^{t}$, i.e. $P_{1}\left(\tau_{k}^{t}\right)=P_{2}\left(\tau_{k}^{t}\right)$, it is possible to show stability for $T_{i}>5.1000$. 
The previous examples show numerically that when the analysis is restricted to the same class of Lyapunov functions, i.e. linear with respect to $\tau_{t}^{k}$, the vector Lyapunov function approach is less conservative than the scalar one. Nevertheless, theoretically speaking, it is very difficult to ensure that the proposed method provides, in general, less conservative results.

The rest of the paper is devoted to the application of the conditions for exponential diagonal $\xi_{k}^{t}$-stability of the impulsive systems (7)-(8), by means of the statements given by Corollaries 1 and 2, in order to solve the sampled-data control problem based on state estimation for system (1)-(3) in a constructive way.

\section{Robust Output-Control Design}

In this section a particular choice for $P_{1}$ and $P_{2}$ is proposed. Then, by means of the statements given by Corollaries 1 and 2, the control gain matrix $K$ and the observer gain matrix $L$ will be found to provide a stabilization of the state dynamics $x$ as well as an estimation $\hat{x}$, i.e. stabilization of the extended state $\xi$ in (5)-(6), for the ideal and uncertain case, respectively.

Thus, the following proposition gives a solution to the sampled-data control problem based on state estimation for the uncertain linear sampled-data system, i.e. $f \neq 0$.

Proposition 1. Consider that $P_{1}$ and $P_{2}$ have the following structure for all $\tau_{k}^{t} \in\left[0, T_{i}\right], i=k \in \mathbb{N}$

$$
P_{1}\left(\tau_{k}^{t}\right)=\frac{\tau_{k}^{t} P_{11}+\left(T_{i}-\tau_{k}^{t}\right) P_{12}}{T_{i}}, P_{2}\left(\tau_{k}^{t}\right)=P_{21}+\tau_{k}^{t} P_{22},
$$

where $P_{11}=\operatorname{diag}\left(P_{11}^{-(1)}, P_{11}^{-(2)}, P_{11}^{-(3)}, P_{11}^{-(3)}\right), P_{12}=\operatorname{diag}\left(P_{12}^{-(1)}, P_{12}^{-(2)}, \delta I_{n}, \delta I_{n}\right)$, with a fixed $\delta>0, P_{1 l}^{(q)}=$ $\left(P_{1 l}^{(q)}\right)^{T}>0$, and $P_{2 l}=P_{2 l}^{T}>0$, for $l=1,2$, and $q=\overline{1,3}$. If there exist matrices $P_{11}^{(q)}, P_{2 l}=P_{2 l}^{T}>0, Y_{K}$ and $L$, for $l=1,2$, and $q=\overline{1,3}$, such that the following matrix inequalities

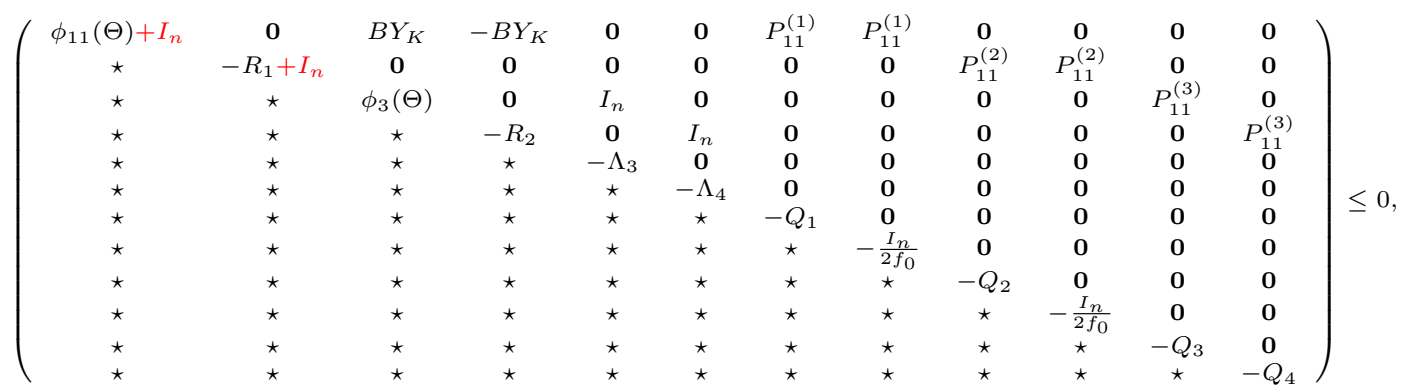

$$
\begin{aligned}
& \left(\begin{array}{ccccccccc|c}
\phi_{21}(\Theta)+I_{n} & \mathbf{0} & B Y_{K} & -B Y_{K} & \mathbf{0} & \mathbf{0} & P_{12}^{(1)} & P_{12}^{(1)} & P_{12}^{(1)} \\
\star & -R_{3}+I_{n} & \mathbf{0} & \mathbf{0} & \mathbf{0} & \mathbf{0} & \mathbf{0} & \mathbf{0} & \mathbf{0} \\
\star & \star & \phi_{3}(\Theta) & \mathbf{0} & \delta P_{11}^{(3)} & \mathbf{0} & \mathbf{0} & \mathbf{0} & \mathbf{0} \\
\star & \star & \star & -R_{4} & \mathbf{0} & \delta P_{11}^{(3)} & \mathbf{0} & \mathbf{0} & \mathbf{0} & \\
\star & \star & \star & \star & -\Lambda_{3} & \mathbf{0} & \mathbf{0} & \mathbf{0} & \mathbf{0} & \mathcal{P} \\
\star & \star & \star & \star & \star & -\Lambda_{4} & \mathbf{0} & \mathbf{0} & \mathbf{0} & \\
\star & \star & \star & \star & \star & \star & -Q_{1} & \mathbf{0} & \mathbf{0} & \\
\star & \star & \star & \star & \star & \star & \star & -\frac{I_{n}}{2 f_{0}} & \mathbf{0} & \\
\star & \star & \star & \star & \star & \star & \star & \star & -\Theta P_{11}^{(1)} & \mathcal{Q}
\end{array}\right) \\
& \mathcal{P}=\left(\begin{array}{cccc}
\mathbf{0}^{T} & P_{12}^{(2)} & \mathbf{0}^{T} & \mathbf{0}^{T} \\
\mathbf{0}^{T} & P_{12}^{(2)} & \mathbf{0}^{T} & \mathbf{0}^{T} \\
\mathbf{0}^{T} & P_{12}^{(2)} & \mathbf{0}^{T} & \mathbf{0}^{T} \\
\mathbf{0}^{T} & \mathbf{0}^{T} & P_{11}^{(3)} & \mathbf{0}^{T} \\
\mathbf{0}^{T} & \mathbf{0}^{T} & \mathbf{0}^{T} & P_{11}^{(3)}
\end{array}\right)^{T}, \mathcal{Q}=\left(\begin{array}{cccccc}
-Q_{2} & \mathbf{0} & \mathbf{0} & \mathbf{0} & \mathbf{0} \\
\star & -\frac{I_{n}}{2 f_{0}} & \mathbf{0} & \mathbf{0} & \mathbf{0} \\
\star & \star & -\Theta P_{11}^{(2)} & \mathbf{0} & \mathbf{0} \\
\star & \star & \star & -Q_{3} & \mathbf{0} \\
\star & \star & \star & \star & -Q_{4}
\end{array}\right), \\
& \left(\begin{array}{cc}
R_{1}+\phi_{12}(\Theta)-L C-C^{T} L^{T} & I_{n} \\
I_{n} & -\bar{\Lambda}_{1}
\end{array}\right) \leq 0,\left(\begin{array}{cc}
R_{2}+\phi_{3}(\Theta) & P_{11}^{(3)} \\
P_{11}^{(3)} & -\bar{\Lambda}_{1}
\end{array}\right) \leq 0, \\
& \left(\begin{array}{cc}
R_{3}+\phi_{22}(\Theta)-L C-C^{T} L^{T} & I_{n} \\
I_{n} & -\bar{\Lambda}_{2}
\end{array}\right) \leq 0,\left(\begin{array}{cc}
R_{4}+\phi_{3}(\Theta) & P_{11}^{(3)} \\
P_{11}^{(3)} & -\bar{\Lambda}_{2}
\end{array}\right) \leq 0, \quad \phi(\Theta) \leq 0,
\end{aligned}
$$


$w_{i t h}^{2}$

$$
\begin{gathered}
\phi_{11}(\Theta)=A P_{11}^{(1)}+P_{11}^{(1)} A^{T}+\frac{P_{11}^{(1)}}{\Theta}-2 P_{11}^{(1)}+\Theta P_{12}^{(1)}, \\
\phi_{12}(\Theta)=A P_{11}^{(2)}+P_{11}^{(2)} A^{T}+\frac{P_{11}^{(2)}}{\Theta}-2 P_{12}^{(2)}-\Theta P_{12}^{(2)}, \\
\phi_{21}(\Theta)=A P_{12}^{(1)}+P_{12}^{(1)} A^{T}-\frac{P_{12}^{(1)}}{\Theta} \\
\phi_{22}(\Theta)=A P_{12}^{(2)}+P_{12}^{(2)} A^{T}-\frac{P_{12}^{(2)}}{\Theta} \\
\phi_{3}(\Theta)=\frac{P_{11}^{(3)}}{\Theta}-2 P_{11}^{(3)}+\frac{\Theta I_{n}}{\delta} \\
\phi(\Theta)=I_{\xi}^{T} P_{21} I_{\xi}-P_{21}-\Theta P_{22}+Q_{5},
\end{gathered}
$$

hold for the finite open set $\Theta \in\left\{0, \frac{c_{2}}{c_{5}} \alpha\right\}, Q=Q^{T}=\operatorname{diag}\left(Q_{1}^{-1}, Q_{2}^{-1}, Q_{3}^{-1}, Q_{4}^{-1}, Q_{5}\right)>0, \Lambda=\Lambda^{T}=\operatorname{diag}\left(I_{n}, I_{n}, \Lambda_{3}, \Lambda_{4}\right)$, some $\bar{\Lambda}_{l}=\bar{\Lambda}_{l}^{T}>0, R_{j}>0$, for $l=1,2$, and $j=\overline{1,4}$, respectively, and $|f(x)|^{2} \leq f_{0}|x|^{2}$; and the constraints (16)(17) also hold with $c_{1}=\lambda_{\min }\left(P_{11}\right), c_{2}=\lambda_{\max }\left(P_{12}\right), c_{3}=\lambda_{\min }\left(P_{21}\right), c_{4}=\lambda_{\max }\left(P_{21}+T_{\max } P_{22}\right)$ and $c_{5}=\lambda_{\min }(Q)$; then the system (7)-(8) is $\mathrm{ED} \xi_{k}^{t}-\mathrm{S}$ for any sequence $\left\{T_{i}\right\}_{i \in \mathbb{N}}$ such that $T_{i} \in\left(0, \frac{c_{2}}{c_{5}} \alpha\right]$ with $K=Y_{K} P_{11}^{-(3)}$ and $L$ solution of (23)-(26).

Now, the following proposition gives a solution to the sampled-data control problem based on state estimation for the ideal linear sampled-data system, i.e. $f=0$.

Proposition 2. Consider that $P_{1}$ and $P_{2}$ have the following structure for all $\tau_{k}^{t} \in\left[0, T_{i}\right], i=k \in \mathbb{N}$

$$
P_{1}\left(\tau_{k}^{t}\right)=\frac{\tau_{k}^{t} P_{11}+\left(T_{i}-\tau_{k}^{t}\right) P_{12}}{T_{i}}, P_{2}\left(\tau_{k}^{t}\right)=P_{21}+\tau_{k}^{t} P_{22},
$$

where $P_{11}=\operatorname{diag}\left(P_{11}^{-(1)}, P_{11}^{-(2)}, P_{11}^{-(3)}, P_{11}^{-(3)}\right), P_{12}=\operatorname{diag}\left(P_{12}^{-(1)}, P_{12}^{-(2)}, \delta I_{n}, \delta I_{n}\right)$, with a fixed $\delta>0, P_{1 l}^{(q)}=$ $\left(P_{1 l}^{(q)}\right)^{T}>0$, and $P_{2 l}=P_{2 l}^{T}>0$, for $l=1,2$, and $q=\overline{1,3}$. If there exist matrices $P_{11}^{(q)}, P_{2 l}=P_{2 l}^{T}>0, Y_{K}$ and $L$, for $l=1,2$, and $q=\overline{1,3}$, such that the following matrix inequalities

$$
\left(\begin{array}{cccccccc}
\phi_{11}(\Theta) & \mathbf{0} & B Y_{K} & -B Y_{K} & P_{11}^{(1)} & \mathbf{0} & \mathbf{0} & \mathbf{0} \\
\star & -R_{1} & \mathbf{0} & \mathbf{0} & \mathbf{0} & P_{11}^{(2)} & \mathbf{0} & \mathbf{0} \\
\star & \star & \phi_{3}(\Theta) & \mathbf{0} & \mathbf{0} & \mathbf{0} & P_{11}^{(3)} & \mathbf{0} \\
\star & \star & \star & -R_{2} & \mathbf{0} & \mathbf{0} & \mathbf{0} & P_{11}^{(3)} \\
\star & \star & \star & \star & -Q_{1} & \mathbf{0} & \mathbf{0} & \mathbf{0} \\
\star & \star & \star & \star & \star & -Q_{2} & \mathbf{0} & \mathbf{0} \\
\star & \star & \star & \star & \star & \star & -Q_{3} & \mathbf{0} \\
\star & \star & \star & \star & \star & \star & \star & -Q_{4}
\end{array}\right) \leq 0,
$$

$$
\left.\begin{array}{cccccccccc}
\phi_{21}(\Theta) & \mathbf{0} & B Y_{K} & -B Y_{K} & P_{12}^{(1)} & P_{12}^{(1)} & \mathbf{0} & \mathbf{0} & \mathbf{0} & \mathbf{0} \\
\star & -R_{3} & \mathbf{0} & \mathbf{0} & \mathbf{0} & \mathbf{0} & P_{12}^{(2)} & P_{12}^{(2)} & \mathbf{0} & \mathbf{0} \\
\star & \star & \phi_{3}(\Theta) & \mathbf{0} & \mathbf{0} & \mathbf{0} & \mathbf{0} & \mathbf{0} & P_{11}^{(3)} & \mathbf{0} \\
\star & \star & \star & -R_{4} & \mathbf{0} & \mathbf{0} & \mathbf{0} & \mathbf{0} & \mathbf{0} & P_{11}^{(3)} \\
\star & \star & \star & \star & -Q_{1} & \mathbf{0} & \mathbf{0} & \mathbf{0} & \mathbf{0} & \mathbf{0} \\
\star & \star & \star & \star & \star & -\Theta P_{11}^{(1)} & \mathbf{0} & \mathbf{0} & \mathbf{0} & \mathbf{0} \\
\star & \star & \star & \star & \star & \star & -Q_{2} & \mathbf{0} & \mathbf{0} & \mathbf{0} \\
\star & \star & \star & \star & \star & \star & \star & -\Theta P_{11}^{(2)} & \mathbf{0} & \mathbf{0} \\
\star & \star & \star & \star & \star & \star & \star & \star & -Q_{3} & \mathbf{0} \\
\star & \star & \star & \star & \star & \star & \star & \star & \star & -Q_{4}
\end{array}\right) \leq 0,
$$

${ }^{2}$ The variables are $P_{11}^{(1)}, P_{11}^{(2)}, P_{11}^{(3)}, P_{12}^{(1)}, P_{12}^{(2)} P_{21}, P_{22}, Y_{K}$ and $L$. The matrices $Q_{i}, \Lambda_{k}, \bar{\Lambda}_{l}$ and $R_{j}$, for $i=\overline{1,5}, k=3,4, l=1,2$, and $i=\overline{1,4}$, respectively, can be declared as variables or fixed values. 


$$
\left(\begin{array}{cc}
R_{3}+\phi_{22}(\Theta)-L C-C^{T} L^{T} & I_{n} \\
I_{n} & -\bar{\Lambda}_{2}
\end{array}\right) \leq 0,\left(\begin{array}{cc}
R_{4}+\phi_{3}(\Theta) & P_{11}^{(3)} \\
P_{11}^{(3)} & -\bar{\Lambda}_{2}
\end{array}\right) \leq 0, \quad \phi(\Theta) \leq 0
$$

with $^{3}$

$$
\begin{gathered}
\phi_{11}(\Theta)=A P_{11}^{(1)}+P_{11}^{(1)} A^{T}+\frac{P_{11}^{(1)}}{\Theta}-2 P_{11}^{(1)}+\Theta P_{12}^{(1)} \\
\phi_{12}(\Theta)=A P_{11}^{(2)}+P_{11}^{(2)} A^{T}+\frac{P_{11}^{(2)}}{\Theta}-2 P_{12}^{(2)}-\Theta P_{12}^{(2)} \\
\phi_{21}(\Theta)=A P_{12}^{(1)}+P_{12}^{(1)} A^{T}-\frac{P_{12}^{(1)}}{\Theta} \\
\phi_{22}(\Theta)=A P_{12}^{(2)}+P_{12}^{(2)} A^{T}-\frac{P_{12}^{(2)}}{\Theta} \\
\phi_{3}(\Theta)=\frac{P_{11}^{(3)}}{\Theta}-2 P_{11}^{(3)}+\frac{\Theta I_{n}}{\delta} \\
\phi(\Theta)=I_{\xi}^{T} P_{21} I_{\xi}-P_{21}-\Theta P_{22}+Q_{5}
\end{gathered}
$$

hold for the finite open set $\Theta \in\left\{0, \frac{c_{2}}{c_{5}} \alpha\right\}, Q=Q^{T}=\operatorname{diag}\left(Q_{1}^{-1}, Q_{2}^{-1}, Q_{3}^{-1}, Q_{4}^{-1}, Q_{5}\right)>0$, some $\bar{\Lambda}_{l}=\bar{\Lambda}_{l}^{T}>0, R_{j}>$ 0 , for $l=1,2$, and $j=\overline{1,4}$, respectively, and constraints (16)-(17) also hold with $c_{1}=\lambda_{\min }\left(P_{11}\right), c_{2}=\lambda_{\max }\left(P_{12}\right)$, $c_{3}=\lambda_{\min }\left(P_{21}\right), c_{4}=\lambda_{\max }\left(P_{21}+T_{\max } P_{22}\right)$ and $c_{5}=\lambda_{\min }(Q)$; then the system $(7)-(8)$ is $\mathrm{ED} \xi_{k}^{t}-\mathrm{S}$ for any sequence $\left\{T_{i}\right\}_{i \in \mathbb{N}}$ such that $T_{i} \in\left(0, \frac{c_{2}}{c_{5}} \alpha\right]$ with $K=Y_{K} P_{11}^{-(3)}$ and $L$ solution of $(27)-(30)$.

Remark 2. Propositions 1 and 2 provide a particular way to solve the proposed problem, i.e. find the control gain matrix $K$ and the gain matrix $L$ such that the system (5)-(6) is exponentially stable for the ideal case and also for the uncertain case.

Numerical Aspects: In order to solve the matrix inequalities provided by Propositions 1 and 2 , one may use a bisection-like approach using SeDuMi solver among YALMIP in Matlab (see e.g. [20] and [30]) on the variable $\Theta \in\left\{0, \frac{c_{2}}{c_{5}} \alpha\right\}$. Providing some initialization values, the bisection method is used to establish the maximum value of $\Theta$ that satisfies the corresponding matrix inequalities, and in turn, compute the constants $c_{1}, c_{2}, c_{3}, c_{4}$ and $c_{5}$ that hold constraints (16)-(17). Note that for fixed $\Theta, \delta$ and $f_{0}$, the matrix inequalities given by Propositions 1 and 2 become LMIs.

Note that a different selection for $P_{1}\left(\tau_{k}^{t}\right)$ and $P_{2}\left(\tau_{k}^{t}\right)$, even for Lyapunov functions with non-quadratic structure, may decrease the conservatism. More complex tools like sum-of-squares [3], looped-functional approach [5], or convex characterizations [4], may be applied to improve the application of this method.

\section{Simulation Results}

\subsection{Ideal Case}

Let us consider system (1)-(2) with $f=0$ and

$$
A=\left(\begin{array}{ll}
0 & 1 \\
0 & 0
\end{array}\right), \quad B=\left(\begin{array}{l}
0 \\
1
\end{array}\right), \quad C=\left(\begin{array}{ll}
1 & 0
\end{array}\right) .
$$

This example represents a double-integrator that has a wide range of applications. Proposition 2 is applied together with a bisection-like approach using SeDuMi solver among YALMIP in Matlab to find a solution for the LMIs, and the corresponding control and observer gains. The simulations have been done in Matlab with the Euler discretization method, sample time equal to 0.001 , and initial conditions $x(0)=(-1,1)^{T}$ and $\hat{x}(0)=(0,0)^{T}$.

Based on Proposition 2, it is possible to show that the impulsive system (5)-(6), with $f=0$, is $E D \xi_{k}^{t}-S$ for all $2.55 \geq T_{i}>0$, i.e. for any sequence $\left\{T_{i}\right\}_{i \in \mathbb{N}}$ such that $T_{i} \in(0,2.55]=\left(T_{\min }, T_{\max }\right]$, there is a set of feasible control

\footnotetext{
${ }^{3}$ The variables are $P_{11}^{(1)}, P_{11}^{(2)}, P_{11}^{(3)}, P_{12}^{(1)}, P_{12}^{(2)} P_{21}, P_{22}, Y_{K}$ and $L$. The matrices $Q_{i}, \bar{\Lambda}_{l}$ and $R_{j}$, for $i=\overline{1,5}, k=3,4, l=1,2$, and $i=\overline{1,4}$, respectively, can be declared as variables or fixed values.
} 
and observer gains. The following feasible results are obtained by fixing different constant values of $T_{i} \in(0,2.55]$, for all $i=0,1,2, \ldots$ :

$$
\begin{aligned}
& T_{i}=0.5 \\
& P_{11}^{(1)}=\left(\begin{array}{ll}
0.0029 & 0.0065 \\
0.0065 & 0.0570
\end{array}\right), P_{11}^{(2)}=\left(\begin{array}{ll}
0.0078 & 0.0072 \\
0.0072 & 0.3891
\end{array}\right), P_{11}^{(3)}=\left(\begin{array}{cc}
0.1854 & 0 \\
0 & 0.0139
\end{array}\right) \\
& P_{12}^{(1)}=\left(\begin{array}{ll}
0.0954 & 0.3702 \\
0.3702 & 1.6643
\end{array}\right), P_{12}^{(2)}=\left(\begin{array}{ll}
0.0095 & 0.0082 \\
0.0082 & 0.0708
\end{array}\right), \delta=1, \\
& P_{21}=\left(\begin{array}{cccccccc}
349.9877 & -0.0226 & -0.0228 & -0.0274 & -125.3336 & 0.0003 & 0.0044 & 0.0033 \\
\star & 349.9973 & -0.0236 & -0.0289 & 0.0040 & -125.3278 & 0.0023 & 0.0027 \\
\star & \star & 349.9921 & -0.0243 & 0.0037 & 0.0003 & -125.3216 & 0.0009 \\
\star & \star & \star & 349.9936 & 0.0021 & 0.0023 & -0.0001 & -125.3348 \\
\star & \star & \star & \star & 234.3587 & 0.0027 & -0.0011 & 0.0006 \\
\star & \star & \star & \star & \star & 234.3513 & 0.0006 & 0.0012 \\
\star & \star & \star & \star & \star & \star & 234.3518 & 0.0019 \\
\star & \star & \star & \star & \star & \star & \star & 234.3589
\end{array}\right) \\
& P_{22}=\left(\begin{array}{cccccccc}
581.5497 & -0.0112 & -0.0188 & -0.0164 & 118.6709 & 0.0067 & 0.0032 & 0.0036 \\
\star & 581.5659 & -0.0187 & -0.0145 & 0.0089 & 118.6656 & 0.0036 & 0.0010 \\
\star & \star & 581.5730 & -0.0201 & 0.0076 & 0.0032 & 118.6649 & 0.0064 \\
\star & \star & \star & 581.5502 & 0.0061 & 0.0029 & 0.0040 & 118.6759 \\
\star & \star & \star & \star & 378.7268 & 0.0040 & -0.0033 & 0.0037 \\
\star & \star & \star & \star & \star & 378.7241 & 0.0003 & 0.0023 \\
\star & \star & \star & \star & \star & \star & 378.7310 & 0.0028 \\
\star & \star & \star & \star & \star & \star & \star & 0.0028
\end{array}\right), \\
& Y_{K}=\left(\begin{array}{ll}
-0.0062 & -0.0062
\end{array}\right), L=\left(\begin{array}{c}
254.4294 \\
33.8006
\end{array}\right), K=\left(\begin{array}{ll}
-0.0352 & -0.4698
\end{array}\right), \\
& T_{i}=1.0 \\
& P_{11}^{(1)}=\left(\begin{array}{ll}
0.0104 & 0.0036 \\
0.0036 & 0.0309
\end{array}\right), P_{11}^{(2)}=\left(\begin{array}{ll}
0.0294 & 0.0035 \\
0.0035 & 0.1299
\end{array}\right), P_{11}^{(3)}=\left(\begin{array}{cc}
0.0991 & 0 \\
0 & 0.0074
\end{array}\right), \\
& P_{12}^{(1)}=\left(\begin{array}{ll}
0.0672 & 0.0803 \\
0.0803 & 0.2420
\end{array}\right), P_{12}^{(2)}=\left(\begin{array}{ll}
0.0382 & 0.0038 \\
0.0038 & 0.0522
\end{array}\right), \delta=1 \\
& P_{21}=\left(\begin{array}{cccccccc}
70.0528 & -0.0047 & -0.0046 & -0.0044 & -17.5110 & 0.0006 & 0.0008 & 0.0011 \\
\star & 70.0528 & -0.0059 & -0.0051 & 0.0011 & -17.5122 & 0.0013 & 0.0007 \\
\star & \star & 70.0521 & -0.0042 & 0.0006 & 0.0015 & -17.5132 & 0.0006 \\
\star & \star & \star & 70.0531 & 0.0009 & 0.0009 & 0.0013 & -17.5134 \\
\star & \star & \star & \star & 50.8534 & -0.0090 & -0.0089 & -0.0088 \\
\star & \star & \star & \star & \star & 50.8526 & -0.0092 & -0.0087 \\
\star & \star & \star & \star & \star & \star & 50.8557 & -0.0088 \\
\star & \star & \star & \star & \star & \star & \star & 50.8532
\end{array}\right), \\
& P_{22}=\left(\begin{array}{cccccccc}
112.1137 & -0.0019 & -0.0027 & -0.0020 & 11.6581 & 0.0130 & 0.0124 & 0.0123 \\
\star & 112.1119 & -0.0023 & -0.0022 & 0.0115 & 11.6589 & 0.0122 & 0.0126 \\
\star & \star & 112.1162 & -0.0024 & 0.0125 & 0.0112 & 11.6591 & 0.0126 \\
\star & \star & \star & 112.1113 & 0.0117 & 0.0126 & 0.0111 & 11.6583 \\
\star & \star & \star & \star & 69.9258 & 0.0207 & 0.0203 & 0.0200 \\
\star & \star & \star & \star & \star & 69.9272 & 0.0205 & 0.0202 \\
\star & \star & \star & \star & \star & \star & 69.9231 & 0.0201 \\
\star & \star & \star & \star & \star & \star & \star & 69.9274
\end{array}\right) \\
& Y_{K}=\left(\begin{array}{ll}
-0.0079 & -0.0079
\end{array}\right), L=\left(\begin{array}{l}
50.1074 \\
21.1055
\end{array}\right), K=\left(\begin{array}{ll}
-0.0796 & -1.0652
\end{array}\right),
\end{aligned}
$$




$$
\begin{aligned}
& T_{i}=2.0 \\
& P_{11}^{(1)}=\left(\begin{array}{ll}
0.0158 & 0.0014 \\
0.0014 & 0.0281
\end{array}\right), P_{11}^{(2)}=\left(\begin{array}{cc}
0.0308 & -0.0011 \\
-0.0011 & 0.0698
\end{array}\right), P_{11}^{(3)}=\left(\begin{array}{cc}
0.1030 & 0 \\
0 & 0.0077
\end{array}\right), \\
& P_{12}^{(1)}=\left(\begin{array}{ll}
0.0777 & 0.0994 \\
0.0994 & 0.6250
\end{array}\right), P_{12}^{(2)}=\left(\begin{array}{ll}
0.0448 & 0.0015 \\
0.0015 & 0.0570
\end{array}\right), \delta=1 \\
& P_{21}=\left(\begin{array}{cccccccc}
57.8022 & 0.0073 & 0.0074 & 0.0067 & -3.9894 & 0.0022 & 0.0023 & 0.0028 \\
\star & 57.8024 & 0.0070 & 0.0065 & 0.0019 & -3.9884 & 0.0019 & 0.0024 \\
\star & \star & 57.8024 & 0.0065 & 0.0020 & 0.0023 & -3.9879 & 0.0025 \\
\star & \star & \star & 57.8027 & 0.0021 & 0.0022 & 0.0027 & -3.9883 \\
\star & \star & \star & \star & 45.6053 & -0.0002 & -0.0002 & -0.0005 \\
\star & \star & \star & \star & \star & 45.6071 & 0.0002 & 0.0001 \\
\star & \star & \star & \star & \star & \star & 45.6062 & 0.0000 \\
\star & \star & \star & \star & \star & \star & \star & 45.6059
\end{array}\right), \\
& P_{22}=\left(\begin{array}{cccccccc}
72.0850 & 0.0095 & 0.0094 & 0.0094 & 1.4037 & 0.0084 & 0.0086 & 0.0084 \\
\star & 72.0859 & 0.0087 & 0.0096 & 0.0088 & 1.4030 & 0.0090 & 0.0084 \\
\star & \star & 72.0879 & 0.0093 & 0.0087 & 0.0084 & 1.4027 & 0.0085 \\
\star & \star & \star & 72.0864 & 0.0085 & 0.0085 & 0.0082 & 1.4031 \\
\star & \star & \star & \star & 42.9554 & 0.0051 & 0.0049 & 0.0052 \\
\star & \star & \star & \star & \star & 42.9547 & 0.0049 & 0.0053 \\
\star & \star & \star & \star & \star & \star & 42.9544 & 0.0054 \\
\star & \star & \star & \star & \star & \star & \star & 42.9544
\end{array}\right) \\
& Y_{K}=\left(\begin{array}{ll}
-0.0065 & -0.0065
\end{array}\right), L=\left(\begin{array}{c}
46.7717 \\
17.7768
\end{array}\right), K=\left(\begin{array}{ll}
-0.0627 & -0.8393
\end{array}\right) .
\end{aligned}
$$

The trajectories of the system, the state estimation error and the control signal for different values of $T_{i}$ are depicted in Figures 1-4. From Fig. 1 it is clear that the trajectories and the estimation worsen whenever the sampling interval increases. However, the proposed approach is capable of stabilizing $\xi_{k}^{t}$ for a reasonable sampling interval equal to 2 seconds. For the aperiodic case, from Fig. 2 it is clear that the proposed approach is able to deal also with the aperiodic case and the behavior is very similar with any of the values in the set of feasible control and observer gains for the same sequence of $T_{i}$. The state estimation error for the ideal case is depicted in Fig. 3 where the estimation error worsens whenever the sampling interval increases. However, the proposed approach is capable of stabilizing $\xi_{k}^{t}$ for a reasonable sampling interval equal to 2 seconds. Finally, the control signals are shown in Fig. 4 .

\subsection{Uncertain Case}

Let us consider system (1)-(2) with

$$
A=\left(\begin{array}{ll}
0 & 1 \\
0 & 0
\end{array}\right), \quad B=\left(\begin{array}{l}
0 \\
1
\end{array}\right), \quad C=\left(\begin{array}{ll}
1 & 0
\end{array}\right), \quad f(x)=0.1\left(\begin{array}{c}
0 \\
x_{1}+\sin \left(x_{2}\right)
\end{array}\right)
$$

This example represents an uncertain double-integrator where $f(x)$ is a Lipschitz function with $f_{0}=0.1$. Proposition 1 is applied together with a bisection-like approach using SeDuMi solver among YALMIP in Matlab to find a solution for the LMIs, and the corresponding control and observer gains. The simulations have been done in Matlab with the Euler discretization method, sample time equal to 0.001 , and initial conditions $x(0)=(-1,1)^{T}$ and $\hat{x}(0)=(0,0)^{T}$.

Based on Proposition 1, it is possible to show that the impulsive system (5)-(6) is $E D \xi_{k}^{t}-S$ for all $0.30>T_{i}>0$ i.e. for any sequence $\left\{T_{i}\right\}_{i \in \mathbb{N}}$ such that $T_{i} \in(0,0.30]=\left(T_{\min }, T_{\max }\right]$, there is a set of feasible control and observer gains. 

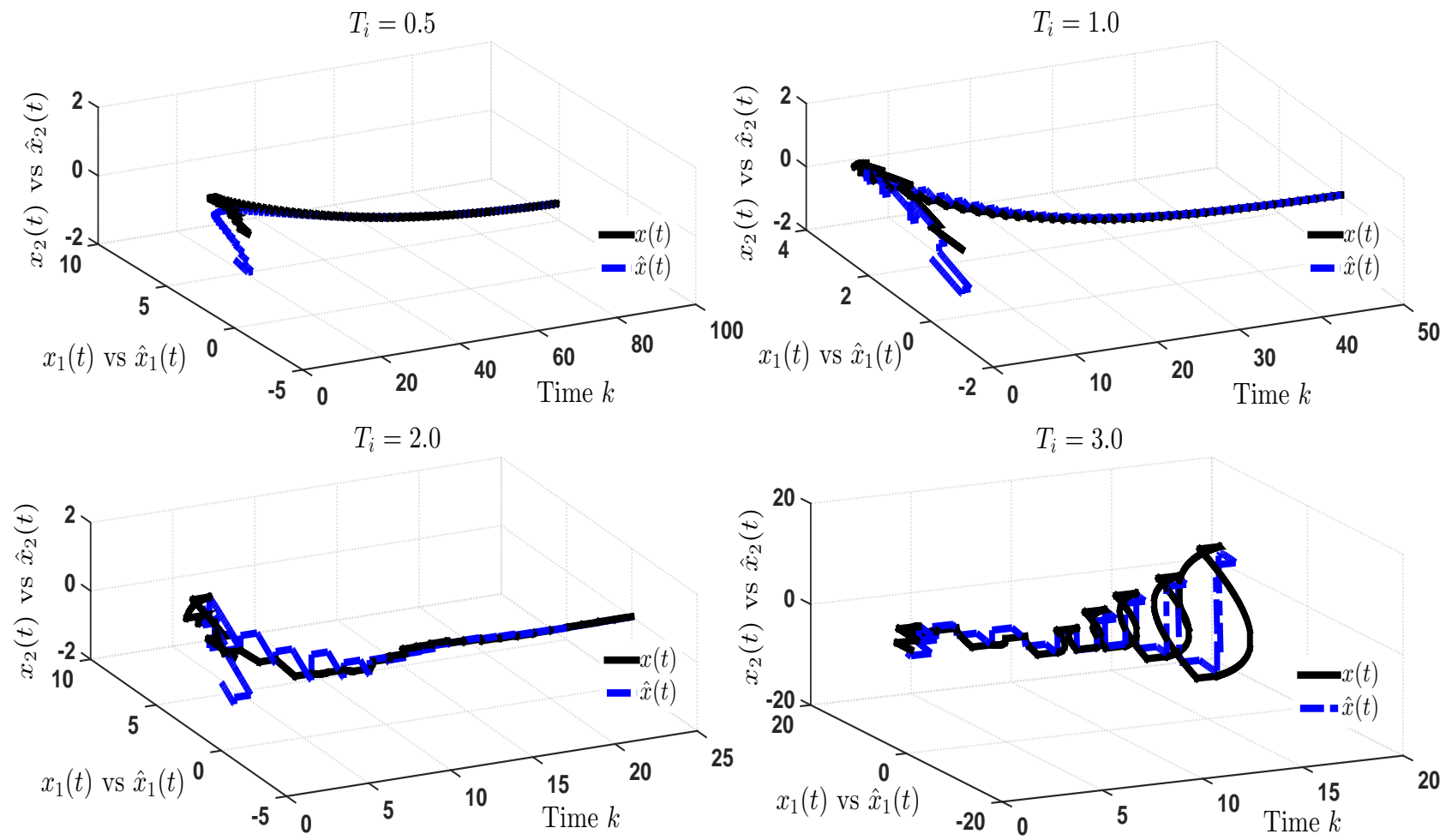

Figure 1: Real and estimated trajectories of the ideal sampled-data system for different values of $T_{i}$

The following feasible results are obtained by fixing different constant values of $T_{i} \in(0,0.30]$, for all $i=0,1,2, \ldots$ :

$$
\begin{aligned}
& T_{i}=0.1 \\
& P_{11}^{(1)}=\left(\begin{array}{ll}
0.0464 & 0.0055 \\
0.0055 & 0.4720
\end{array}\right), P_{11}^{(2)}=\left(\begin{array}{ll}
0.0158 & 0.0296 \\
0.0296 & 4.5343
\end{array}\right), P_{11}^{(3)}=\left(\begin{array}{cc}
0.6860 & -0.0234 \\
-0.0234 & 0.3431
\end{array}\right), \\
& P_{12}^{(1)}=\left(\begin{array}{ll}
0.5672 & 0.0264 \\
0.0264 & 0.4714
\end{array}\right), P_{12}^{(2)}=\left(\begin{array}{cc}
2.7065 & -0.2887 \\
-0.2887 & 2.9879
\end{array}\right), \delta=1, \\
& P_{21}=\left(\begin{array}{cccccccc}
83.0960 & 0.0266 & 0.0266 & 0.0266 & -37.5004 & -0.0060 & -0.0060 & -0.0060 \\
\star & 83.0960 & 0.0266 & 0.0266 & -0.0060 & -37.5004 & -0.0060 & -0.0060 \\
\star & \star & 83.0960 & 0.0266 & -0.0060 & -0.0060 & -37.5004 & -0.0060 \\
\star & \star & \star & 83.0960 & -0.0060 & -0.0060 & -0.0060 & -37.5004 \\
\star & \star & \star & \star & 43.8472 & -0.0198 & -0.0198 & -0.0198 \\
\star & \star & \star & \star & \star & 43.8472 & -0.0198 & -0.0198 \\
\star & \star & \star & \star & \star & \star & 43.8472 & -0.0198 \\
\star & \star & \star & \star & \star & \star & \star & 43.8472
\end{array}\right), \\
& P_{22}=\left(\begin{array}{cccccccc}
127.4672 & 0.9881 & 0.9881 & 0.9881 & 62.8240 & 0.8689 & 0.8689 & 0.8698 \\
\star & 127.4672 & 0.9881 & 0.9881 & 0.8688 & 62.8240 & 0.8689 & 0.8689 \\
\star & \star & 127.4672 & 0.9881 & 0.8689 & 0.8689 & 62.8240 & 0.8689 \\
\star & \star & \star & 127.4672 & 0.8689 & 0.8689 & 0.8689 & 62.8240 \\
\star & \star & \star & \star & 106.2215 & 0.7620 & 0.7619 & 0.7619 \\
\star & \star & \star & \star & \star & 106.2215 & 0.7619 & 0.7619 \\
\star & \star & \star & \star & \star & \star & 106.2215 & 0.7618 \\
\star & \star & \star & \star & \star & \star & \star & 106.2215
\end{array}\right) \\
& Y_{K}=\left(\begin{array}{ll}
-0.9182 & -0.9521
\end{array}\right), L=\left(\begin{array}{l}
2.6175 \\
3.7852
\end{array}\right), K=\left(\begin{array}{ll}
-1.4364 & -2.8729
\end{array}\right),
\end{aligned}
$$


$T_{i} \in[0.5,2.0]$
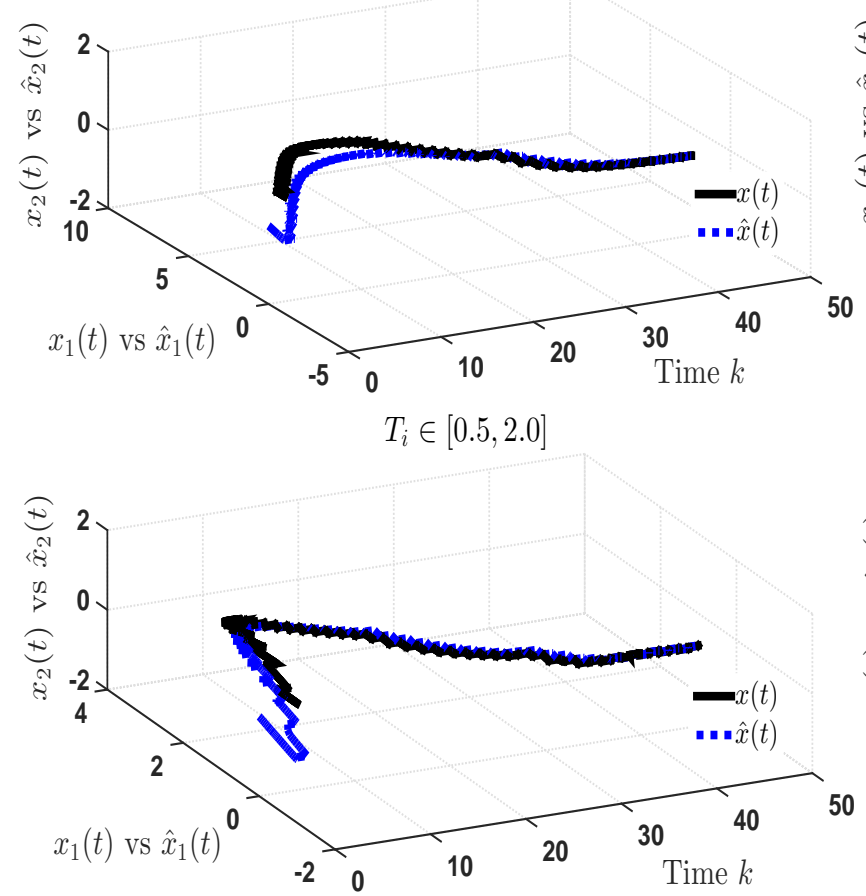

$T_{i} \in[0.5,2.0]$
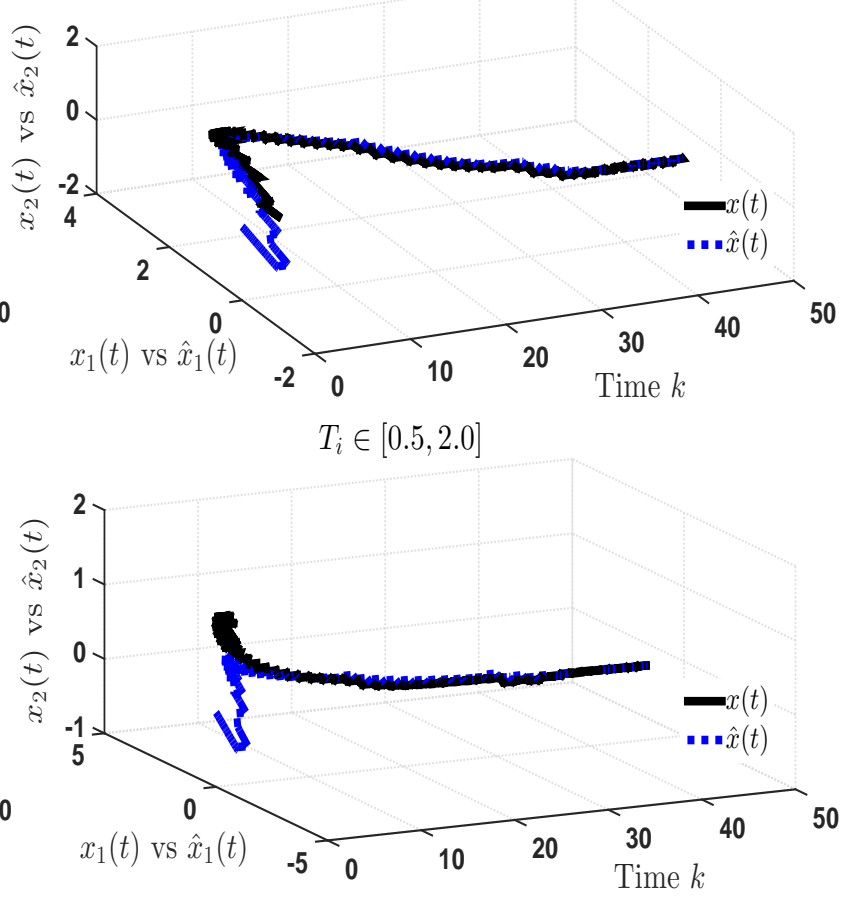

Figure 2: Real and estimated trajectories of the ideal sampled-data system for the aperiodic case $T_{i} \in[0.5,2.0]$ and different values of $K$ and $L$. For a fixed sequence in $T_{i}$, four different matrix gains, in the set of feasible solutions, for $K$ and $L$ were used, i.e. $K=(-0.0352,-0.4698), L=(254.4294,33.8006)^{T}-$ left top; $K=(-0.0796,-1.0652)$, $L=(50.1074,21.1055)^{T}$ - right top; $K=(-0.0771,-1.0328), L=(41.5574,17.6644)^{T}$ - left bottom; $K=(-0.0627,-0.8393)$, $L=(46.7717,17.7768)^{T}$ - right bottom.

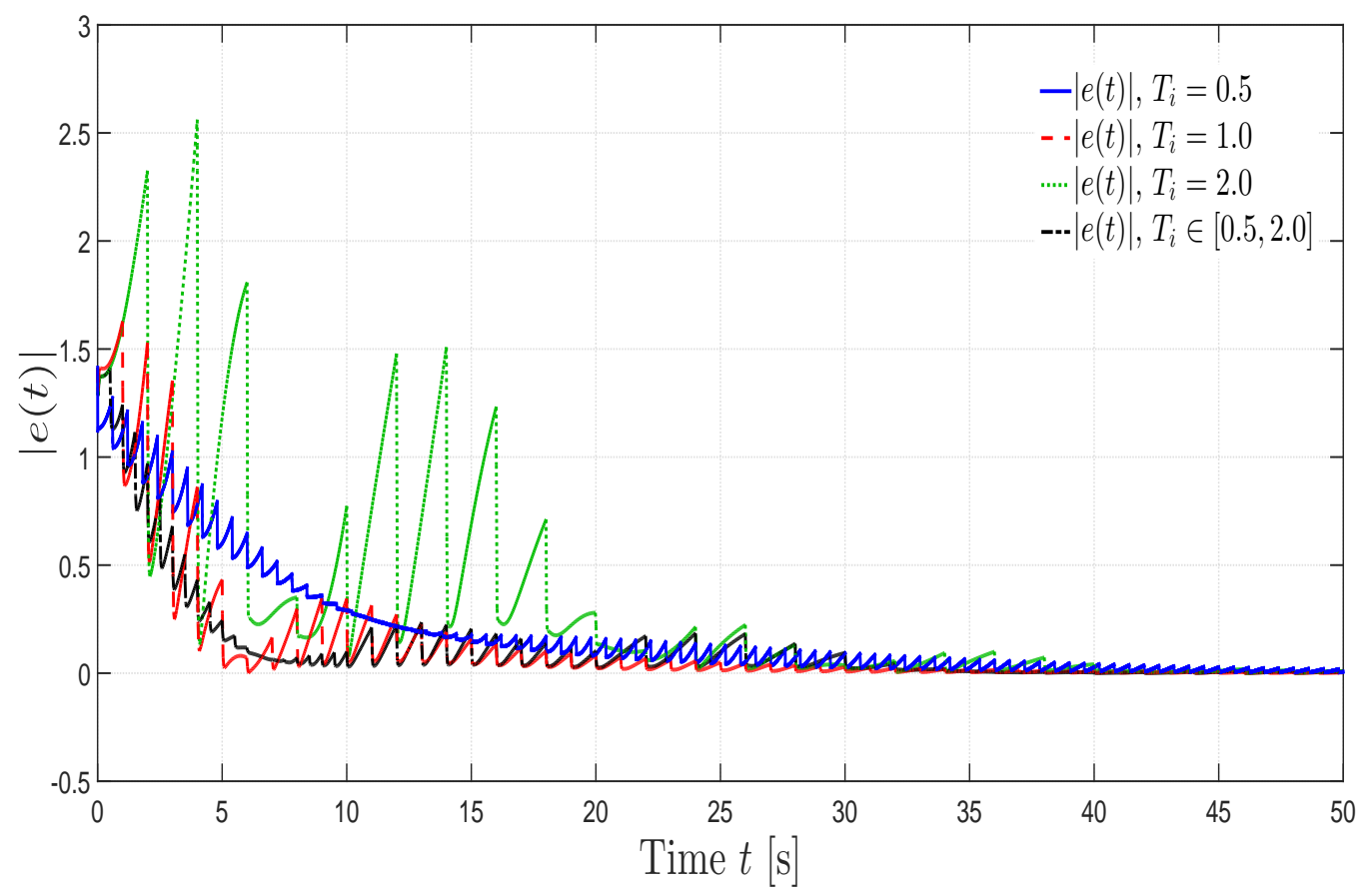

Figure 3: State estimation error of the ideal sampled-data system for different values of $T_{i}$ including the aperiodic case. For the aperiodic case the gains were $K=(-0.0627,-0.8393), L=(46.7717,17.7768)^{T}$ corresponding to $T_{i}=2.0$. 


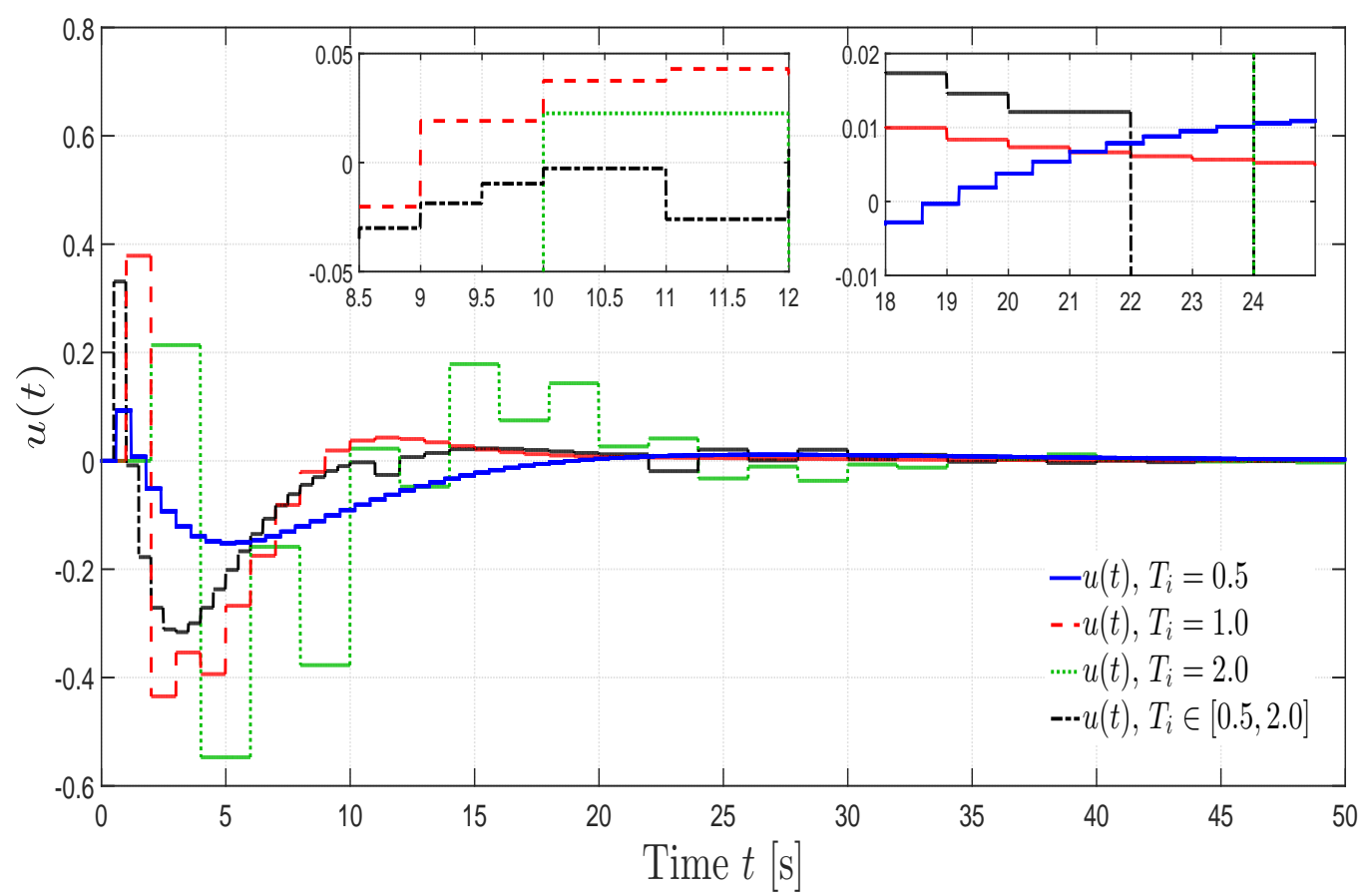

Figure 4: Control signal of the ideal sampled-data system for different values of $T_{i}$ including the aperiodic case. For the aperiodic case the gains were $K=(-0.0627,-0.8393), L=(46.7717,17.7768)^{T}$ corresponding to $T_{i}=2.0$.

$$
\begin{aligned}
& T_{i}=0.2 \\
& P_{11}^{(1)}=\left(\begin{array}{cc}
0.8291 & -0.1799 \\
-0.1799 & 0.9342
\end{array}\right), P_{11}^{(2)}=\left(\begin{array}{ll}
0.0416 & 0.0238 \\
0.0238 & 5.7885
\end{array}\right), P_{11}^{(3)}=\left(\begin{array}{cc}
0.3826 & -0.0076 \\
-0.0076 & 0.1913
\end{array}\right) \\
& P_{12}^{(1)}=\left(\begin{array}{cc}
2.7765 & -0.1098 \\
-0.1098 & 2.1288
\end{array}\right), P_{12}^{(2)}=\left(\begin{array}{cc}
4.3625 & -0.1738 \\
-0.1738 & 5.7290
\end{array}\right), \delta=1, \\
& P_{21}=\left(\begin{array}{cccccccc}
77.3837 & 0.0084 & 0.0084 & 0.0084 & -34.3973 & 0.0002 & 0.0002 & 0.0002 \\
\star & 77.3837 & 0.0084 & 0.0084 & 0.0002 & -34.3973 & 0.0002 & 0.0002 \\
\star & \star & 77.3837 & 0.0084 & 0.0002 & 0.0002 & -34.3973 & 0.0002 \\
\star & \star & \star & 77.3837 & 0.0002 & 0.0002 & 0.0002 & -34.3973 \\
\star & \star & \star & \star & 44.4824 & -0.0124 & -0.0124 & -0.0124 \\
\star & \star & \star & \star & \star & 44.4824 & -0.0124 & -0.0124 \\
\star & \star & \star & \star & \star & \star & 44.4824 & -0.0124 \\
\star & \star & \star & \star & \star & \star & \star & 44.4824
\end{array}\right), \\
& P_{22}=\left(\begin{array}{cccccccc}
123.7642 & 0.8964 & 0.8964 & 0.8964 & 51.1589 & 0.7385 & 0.7385 & 0.7385 \\
\star & 123.7642 & 0.8964 & 0.8964 & 0.7385 & 51.1589 & 0.7385 & 0.7385 \\
\star & \star & 123.7642 & 0.8964 & 0.7385 & 0.7385 & 51.1589 & 0.7385 \\
\star & \star & \star & 123.7642 & 0.7385 & 0.7385 & 0.7385 & 51.1589 \\
\star & \star & \star & \star & 93.4763 & 0.6062 & 0.6062 & 0.6062 \\
\star & \star & \star & \star & \star & 93.4763 & 0.6062 & 0.6062 \\
\star & \star & \star & \star & \star & \star & 93.4763 & 0.6062 \\
\star & \star & \star & \star & \star & \star & \star & 93.4763
\end{array}\right), \\
& Y_{K}=\left(\begin{array}{ll}
-0.1032 & -0.1054
\end{array}\right), L=\left(\begin{array}{l}
2.5540 \\
3.1337
\end{array}\right), K=\left(\begin{array}{ll}
-0.2810 & -0.5620
\end{array}\right),
\end{aligned}
$$




$$
\begin{aligned}
& T_{i}=0.3 \\
& P_{11}^{(1)}=\left(\begin{array}{ll}
0.7794 & 0.1081 \\
0.1081 & 1.3372
\end{array}\right), P_{11}^{(2)}=\left(\begin{array}{ll}
0.0358 & 0.0284 \\
0.0284 & 2.4736
\end{array}\right), P_{11}^{(3)}=\left(\begin{array}{cc}
0.3749 & -0.0046 \\
-0.0046 & 0.3749
\end{array}\right) \\
& P_{12}^{(1)}=\left(\begin{array}{cc}
2.8366 & -0.2920 \\
-0.2920 & 2.5168
\end{array}\right), P_{12}^{(2)}=\left(\begin{array}{cc}
2.7932 & -0.0059 \\
-0.0059 & 3.6565
\end{array}\right), \delta=1, \\
& P_{21}=\left(\begin{array}{cccccccc}
73.0348 & 0.0024 & 0.0024 & 0.0024 & -31.3059 & -0.0001 & -0.0001 & -0.0001 \\
\star & 73.0348 & 0.0024 & 0.0024 & -0.0001 & -31.3059 & -0.0001 & -0.0001 \\
\star & \star & 73.0348 & 0.0024 & -0.0001 & -0.0001 & -31.3059 & -0.0001 \\
\star & \star & \star & 73.0348 & -0.0001 & -0.0001 & -0.0001 & -31.3059 \\
\star & \star & \star & \star & 44.3482 & 0.0003 & 0.0003 & 0.0003 \\
\star & \star & \star & \star & \star & 44.3482 & 0.0003 & 0.0003 \\
\star & \star & \star & \star & \star & \star & 44.3482 & 0.0003 \\
\star & \star & \star & \star & \star & \star & \star & 44.3482
\end{array}\right), \\
& P_{22}=\left(\begin{array}{cccccccc}
118.7790 & 0.0300 & 0.0300 & 0.0300 & 40.8381 & 0.0198 & 0.0198 & 0.0198 \\
\star & 118.7790 & 0.0300 & 0.0300 & 0.0198 & 40.8381 & 0.0198 & 0.0198 \\
\star & \star & 118.7790 & 0.0300 & 0.0198 & 0.0198 & 40.8381 & 0.0198 \\
\star & \star & \star & 118.7790 & 0.0198 & 0.0198 & 0.0198 & 40.8381 \\
\star & \star & \star & \star & 83.9838 & 0.0132 & 0.0132 & 0.0132 \\
\star & \star & \star & \star & \star & 83.9838 & 0.0132 & 0.0132 \\
\star & \star & \star & \star & \star & \star & 83.9838 & 0.0132 \\
\star & \star & \star & \star & \star & \star & \star & 83.9838
\end{array}\right) \\
& Y_{K}=\left(\begin{array}{ll}
-0.1136 & -0.2315
\end{array}\right), L=\left(\begin{array}{l}
2.5714 \\
1.9137
\end{array}\right), K=\left(\begin{array}{ll}
-0.3107 & -0.6213
\end{array}\right) .
\end{aligned}
$$

The trajectories of the system, the state estimation error and the control signal for different values of $T_{i}$ are depicted in Figures 5-8. From Fig. 5 it is clear that for the uncertain case, the trajectories deteriorate, more than the ideal case, whenever the sampling interval increases. The proposed approach is capable of stabilizing $\xi_{k}^{t}$ for a sampling interval less than or equal to 0.30 seconds. For the aperiodic case, from Fig. 6 it is clear that the proposed approach is able to deal also with the aperiodic case and the behavior is very similar with any of the values in the set of feasible control and observer gains for the same sequence of $T_{i}$ but the sampling interval has decreased with respect to the ideal case. The state estimation error for the ideal case is depicted in Fig. 7 where the estimation error deteriorates, more than the ideal case, whenever the sampling interval increases. The proposed approach is capable of stabilizing $\xi_{k}^{t}$ for a sampling interval less than or equal to 0.30 seconds. Finally, the control signals are shown in Fig. 8 .

\section{Conclusions}

In this paper a vector Lyapunov function-based approach, derived by means of a $2 D$ time domain equivalence, for stability of impulsive systems is used for designing a robust output-feedback control for linear sampled-data systems. This approach provides a stability analysis based on LMIs for linear impulsive dynamical systems. Then, it is possible to show that the sampled-data control problem based on state estimation may turn into one of finding conditions for the exponential stability of impulsive systems. Thus, the proposed vector Lyapunov function approach is applied for obtaining stability conditions of the impulsive system, and then, a solution to the robust output-feedback control design problem is derived and expressed in terms of LMIs. Some numerical examples illustrate the feasibility of the proposed approach. The analysis of uncertain sampled-data nonlinear systems is in the scope of the future research. 

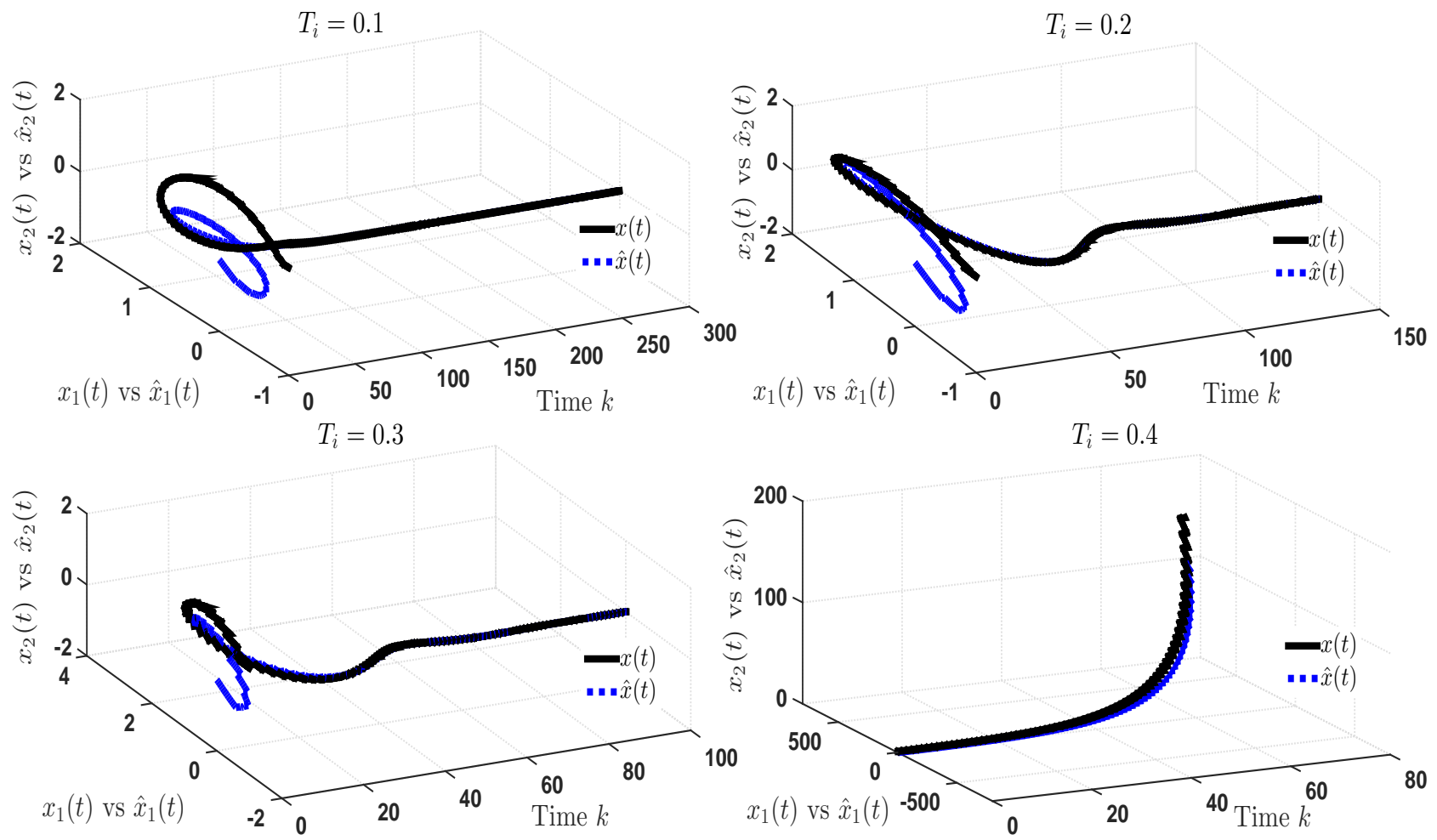

Figure 5: Real and estimated trajectories of the uncertain sampled-data system for different values of $T_{i}$

\section{Appendix}

Proof of Theorem 1: From the divergence definition and inequalities (13), (14) and (15), it follows that

$$
\begin{aligned}
\frac{d V_{1}\left(z_{k}^{t}\right)}{d t} & \leq-c_{5}\left(\left|z_{k}^{t}\right|^{2}+\left|z_{k}^{t_{i+1}}\right|^{2}\right)-V_{2}\left(z_{k+1}^{t_{i+1}}\right)+V_{2}\left(z_{k}^{t_{i+1}}\right), \\
& \leq-\beta V_{1}\left(z_{k}^{t}\right)+\lambda V_{2}\left(z_{k}^{t_{i+1}}\right)-V_{2}\left(z_{k+1}^{t_{i+1}}\right),
\end{aligned}
$$

where $\lambda=1-\frac{c_{5}}{c_{4}}$ and $\beta=\frac{c_{5}}{c_{2}}$. By means of the comparison principle, with respect to the time $t$, from (31), for all $t \in\left[t_{i}, t_{i+1}\right)$, it is obtained that

$$
\begin{aligned}
V_{1}\left(z_{k}^{t}\right) & \leq e^{-\beta\left(t-t_{i}\right)} V_{1}\left(z_{k}^{t_{i}}\right)+\int_{t_{i}}^{t} e^{-\beta(t-\tau)}\left[\lambda V_{2}\left(z_{k}^{t_{i+1}}\right)-V_{2}\left(z_{k+1}^{t_{i+1}}\right)\right] d \tau, \\
& =e^{-\beta\left(t-t_{i}\right)} V_{1}\left(z_{k}^{t_{i}}\right)+\rho_{i}(t)\left(\lambda V_{2}\left(z_{k}^{t_{i+1}}\right)-V_{2}\left(z_{k+1}^{t_{i+1}}\right)\right),
\end{aligned}
$$

where $\rho_{i}(t)=\frac{1-e^{-\beta\left(t-t_{i}\right)}}{\beta}>0$, for all $t \in\left[t_{i}, t_{i+1}\right)$. In order to fulfill the statements given by Definition 1 it is necessary to prove convergence and boundedness. Thus, let us prove each one separately.

1. Convergence. Evaluating (32) for $t=t_{i+1}$, it gives

$$
V_{1}\left(z_{k}^{t_{i+1}}\right) \leq e^{-\beta T_{i}} V_{1}\left(z_{k}^{t_{i}}\right)+\rho_{i}\left(t_{i+1}\right) \lambda V_{2}\left(z_{k}^{t_{i+1}}\right)-\rho_{i}\left(t_{i+1}\right) V_{2}\left(z_{k+1}^{t_{i+1}}\right)
$$



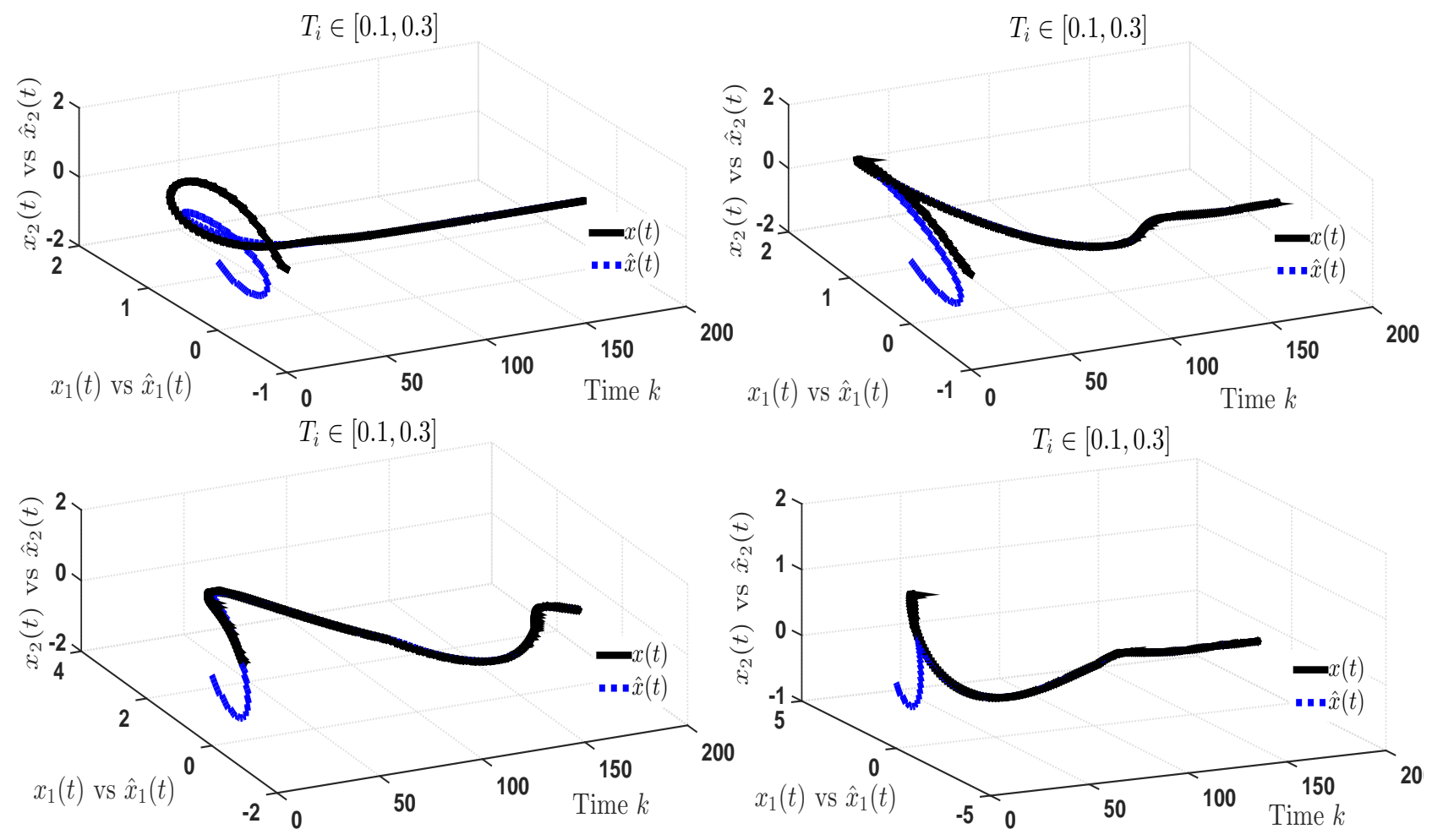

Figure 6: Real and estimated trajectories of the ideal sampled-data system for the aperiodic case $T_{i} \in[0.1,0.3]$ and different values of $K$ and $L$. For a fixed sequence in $T_{i}$, four different matrix gains, in the set of feasible solutions, for $K$ and $L$ were used, i.e. $K=(-1.4364,-2.8729), L=(2.6175,3.7852)^{T}-$ left top; $K=(-0.2810,-0.5620), L=(2.5540,3.1337)^{T}$ - right top; $K=(-0.1013,-0.2025), L=(2.5453,4.7680)^{T}$ - left bottom; $K=(-0.3107,-0.6213), L=(2.5714,1.9137)^{T}$ right bottom.

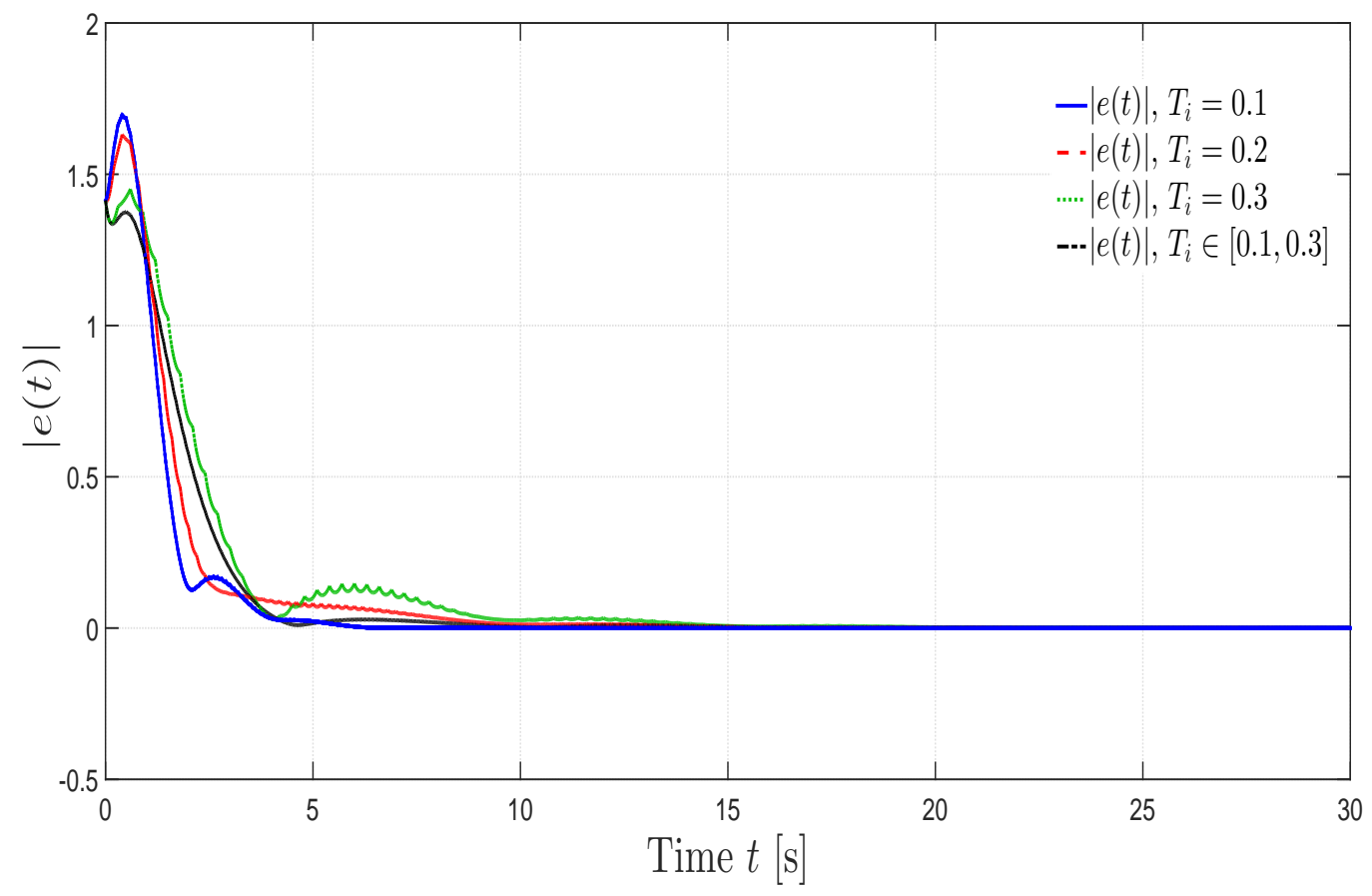

Figure 7: State estimation error of the uncertain sampled-data system for different values of $T_{i}$ including the aperiodic case. For the aperiodic case the gains were $K=(-0.3107,-0.6213), L=(2.5714,1.9137)^{T}$ corresponding to $T_{i}=0.3$. 


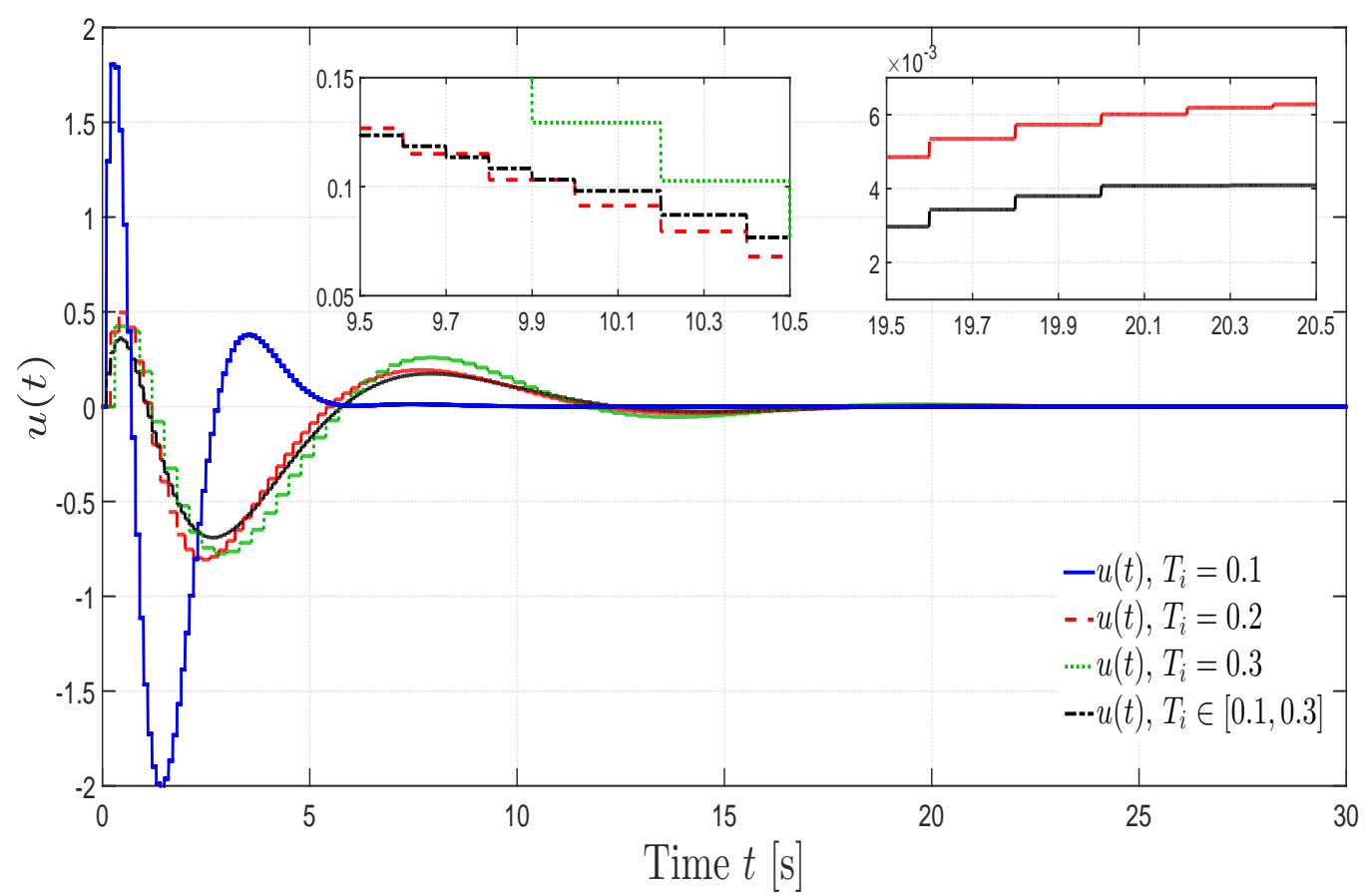

Figure 8: Control signal of the uncertain sampled-data system for different values of $T_{i}$ including the aperiodic case. For the aperiodic case the gains were $K=(-0.3107,-0.6213), L=(2.5714,1.9137)^{T}$ corresponding to $T_{i}=0.3$.

with $\rho_{i}\left(t_{i+1}\right)=\frac{1-e^{-\beta T_{i}}}{\beta}>0$, for all $T_{i} \geq 0$. From the inequalities (13) and (14), it follows that $\forall i=k \in \mathbb{N}$

$$
\begin{aligned}
& \frac{V_{2}\left(z_{k}^{p}\right)}{c_{4}} \leq\left|\xi_{k}^{p}\right|^{2} \leq \frac{V_{1}\left(z_{k}^{p}\right)}{c_{1}}, \forall p=t_{i}, t_{i+1} \\
& \frac{V_{1}\left(z_{k}^{p}\right)}{c_{2}} \leq\left|\xi_{k}^{p}\right|^{2} \leq \frac{V_{2}\left(z_{k}^{p}\right)}{c_{3}}, \forall p=t_{i}, t_{i+1} .
\end{aligned}
$$

From (34), it is given that $\frac{c_{1}}{c_{4}} V_{2}\left(z_{k}^{t_{i+1}}\right) \leq V_{1}\left(z_{k}^{t_{i+1}}\right)$, and therefore from (33), it is obtained that

$$
\rho_{i}\left(t_{i+1}\right) V_{2}\left(z_{k+1}^{t_{i+1}}\right) \leq e^{-\beta T_{i}} V_{1}\left(z_{k}^{t_{i}}\right)+\left(\rho_{i}\left(t_{i+1}\right) \lambda-\frac{c_{1}}{c_{4}}\right) V_{2}\left(z_{k}^{t_{i+1}}\right) .
$$

Let us consider that $c_{4}>c_{5}$, i.e. $\lambda \in(0,1)$. Thus, from (35) and (36) it follows that

$$
V_{2}\left(z_{k+1}^{t_{i+1}}\right) \leq \frac{c_{2} e^{-\beta T_{i}}}{c_{3} \rho_{i}\left(t_{i+1}\right)} V_{2}\left(z_{k}^{t_{i}}\right)+\left(\frac{\rho_{i}\left(t_{i+1}\right) \lambda-\frac{c_{1}}{c_{4}}}{\rho_{i}\left(t_{i+1}\right)}\right) V_{2}\left(z_{k}^{t_{i+1}}\right) .
$$

Note that if the constraint $\rho_{i}\left(t_{i+1}\right) \lambda \leq \frac{c_{1}}{c_{4}}$ holds, then the term depending on $V_{2}\left(z_{k}^{t_{i+1}}\right)$ can be disregarded. In this sense, in order to satisfy such a constraint, recalling that $\beta=\frac{c_{5}}{c_{2}}, \lambda=1-\frac{c_{5}}{c_{4}}$ and $\rho_{i}\left(t_{i+1}\right)=\frac{1-e^{-\beta T_{i}}}{\beta}$, the following condition is founded

$$
\begin{aligned}
c_{2}\left(1-e^{-\beta T_{i}}\right)\left(c_{4}-c_{5}\right) & \leq c_{1} c_{5}, \\
\left(1-e^{-\beta T_{i}}\right) & \leq \frac{c_{1} c_{5}}{c_{2}\left(c_{4}-c_{5}\right)} .
\end{aligned}
$$


Then, it is clear that if $c_{2}\left(c_{4}-c_{5}\right) \leq c_{1} c_{5}$ holds then $\rho_{i}\left(t_{i+1}\right) \lambda \leq \frac{c_{1}}{c_{4}}$ is trivially satisfied. Otherwise

$$
e^{-\beta T_{i}} \geq \frac{c_{2}\left(c_{4}-c_{5}\right)-c_{1} c_{5}}{c_{2}\left(c_{4}-c_{5}\right)} \Leftrightarrow T_{i} \leq \frac{c_{2}}{c_{5}} \alpha
$$

where $\alpha=-\ln \left[\frac{c_{2}\left(c_{4}-c_{5}\right)-c_{1} c_{5}}{c_{2}\left(c_{4}-c_{5}\right)}\right]>0$, for all $c_{2}\left(c_{4}-c_{5}\right)>c_{1} c_{5}$. Note that these two possibilities, i.e. $c_{2}\left(c_{4}-c_{5}\right) \leq$ $c_{1} c_{5}$ or $T_{i} \leq \frac{c_{2}}{c_{5}} \alpha$, are represented by (16) in Theorem 1. Therefore, if one of them is satisfied, from (37) it is obtained that

$$
V_{2}\left(z_{k+1}^{t_{i+1}}\right) \leq \frac{c_{2} e^{-\beta T_{i}}}{c_{3} \rho_{i}\left(t_{i+1}\right)} V_{2}\left(z_{k}^{t_{i}}\right) .
$$

Then, by induction, it follows that

$$
V_{2}\left(z_{k+1}^{t_{i+1}}\right) \leq\left(\frac{c_{2} e^{-\beta T_{i}}}{c_{3} \rho_{i}\left(t_{i+1}\right)}\right)^{k+1} V_{2}\left(z_{0}^{0}\right)
$$

Hence, (38) decreases if the following condition holds

$$
\begin{aligned}
\frac{c_{2} e^{-\beta T_{i}}}{c_{3} \rho_{i}\left(t_{i+1}\right)} & \leq 1-\varepsilon \\
c_{5} e^{-\beta T_{i}} & \leq c_{3}(1-\varepsilon)\left(1-e^{-\beta T_{i}}\right), \\
e^{-\beta T_{i}} & \leq \frac{c_{3}(1-\varepsilon)}{c_{5}+c_{3}(1-\varepsilon)} \Leftrightarrow T_{i} \geq \frac{c_{2}}{c_{5}} \gamma
\end{aligned}
$$

which is the same that (17), with $\gamma=-\ln \left[\frac{c_{3}(1-\varepsilon)}{c_{5}+c_{3}(1-\varepsilon)}\right]$. Then, from (34), (35), and (38), it follows that $\forall i=k \in \mathbb{N}$

$$
\left|\xi_{k+1}^{t_{i+1}}\right|^{2} \leq c \kappa_{1}^{k+1}\left|\xi_{0}^{0}\right|^{2}
$$

with $c=\frac{c_{4}}{c_{3}}>0$ and $0<\kappa_{1}=\frac{c_{5}}{c_{3}\left(1-e^{-\gamma}\right)}<1-\varepsilon$, for some small positive $\varepsilon$. Thus, the trajectories of system (7)-(8) are convergent under the constraints $c_{4}>c_{5}, c_{2}\left(c_{4}-c_{5}\right) \leq c_{1} c_{5}$ or $T_{i} \leq \frac{c_{2}}{c_{5}} \alpha$, and $T_{i} \geq \frac{c_{2}}{c_{5}} \gamma$, i.e. eq. (9) from Definition 1 is obtained. Now, let us take into account that $c_{5} \geq c_{4}$, i.e. $\lambda \leq 0$. Therefore, from (36), it follows that the term depended on $V_{2}\left(z_{k}^{t_{i+1}}\right)$ can be disregarded, then one gets (38) and just under condition $T_{i} \geq \frac{c_{2}}{c_{5}} \gamma$ convergence is obtained. Thus, it is concluded that the trajectories of system (7)-(8) are convergent under constraints (16)-(17) if $c_{4}>c_{5}$, or only under (17) if $c_{5} \geq c_{4}$ holds. In order to complete the proof, let us prove boundedness between the impulses, i.e. $\left|\xi_{k}^{t}\right|^{2} \leq \kappa_{2}\left|\xi_{k}^{t_{i}}\right|^{2}$ for all $t \in\left[t_{i}, t_{i+1}\right)$.

2. Boundedness. From (32), it is given that

$$
V_{1}\left(z_{k}^{t}\right) \leq e^{-\beta\left(t-t_{i}\right)} V_{1}\left(z_{k}^{t_{i}}\right)+\rho_{i}\left(t_{i+1}\right) \lambda V_{2}\left(z_{k}^{t_{i+1}}\right) .
$$

Let us consider the case $c_{5} \geq c_{4}$, i.e. $\lambda \leq 0$. Therefore, from (39), it follows that $V_{1}\left(z_{k}^{t}\right) \leq e^{-\beta\left(t-t_{i}\right)} V_{1}\left(z_{k}^{t_{i}}\right)$, $\forall i=k \in \mathbb{N}$, and boundedness is given, i.e.

$$
\left|\xi_{k}^{t}\right|^{2} \leq \kappa_{2}\left|\xi_{k}^{t_{i}}\right|^{2}, \forall t \in\left[t_{i}, t_{i+1}\right)
$$

with $\kappa_{2}=\frac{c_{2}}{c_{1}}$. Finally, for the case $c_{4}>c_{5}$, i.e. $\lambda \in(0,1)$, from (39) and evaluating $t=t_{i+1}$, one gets

$$
\begin{aligned}
\frac{c_{1}}{c_{4}} V_{2}\left(z_{k}^{t_{i+1}}\right) & \leq e^{-\beta T_{i}} V_{1}\left(z_{k}^{t_{i}}\right)+\rho_{i}\left(t_{i+1}\right) \lambda V_{2}\left(z_{k}^{t_{i+1}}\right), \\
V_{2}\left(z_{k}^{t_{i+1}}\right) & \leq \frac{e^{-\beta T_{i}}}{\left(\frac{c_{1}}{c_{4}}-\rho_{i}\left(t_{i+1}\right) \lambda\right)} V_{1}\left(z_{k}^{t_{i}}\right) .
\end{aligned}
$$

Note that $\rho_{i}\left(t_{i+1}\right) \lambda<\frac{c_{1}}{c_{4}}$ has to hold in order to satisfy inequality (40). However, as it was previously described, if $c_{2}\left(c_{4}-c_{5}\right) \leq c_{1} c_{5}$ holds, $\rho_{i}\left(t_{i+1}\right) \lambda<\frac{c_{1}}{c_{4}}$ is trivially satisfied, otherwise $T_{i}$ should be less than or equal to $\frac{c_{2}}{c_{5}} \alpha$, i.e. $T_{i} \leq \frac{c_{2}}{c_{5}} \alpha$ with $\alpha=-\ln \left[\frac{c_{2}\left(c_{4}-c_{5}\right)-c_{1} c_{5}}{c_{2}\left(c_{4}-c_{5}\right)}\right]>0$, for all $c_{2}\left(c_{4}-c_{5}\right)>c_{1} c_{5}$. Thus, applying $(40)$ 
in (39), it is given that

$$
\begin{aligned}
V_{1}\left(z_{k}^{t}\right) & \leq\left[e^{-\beta\left(t-t_{i}\right)}+\frac{\rho_{i}\left(t_{i+1}\right) \lambda e^{-\beta T_{i}}}{\left(\frac{c_{1}}{c_{4}}-\rho_{i}\left(t_{i+1}\right) \lambda\right)}\right] V_{1}\left(z_{k}^{t_{i}}\right), \\
& \leq\left[\frac{c_{5}\left(c_{1}-c_{4} \rho_{i}\left(t_{i+1}\right) \lambda\right)+c_{2} c_{4}}{c_{5}\left(c_{1}-c_{4} \rho_{i}\left(t_{i+1}\right) \lambda\right)}\right] V_{1}\left(z_{k}^{t_{i}}\right) .
\end{aligned}
$$

Therefore, from (41), boundedness is obtained, i.e. $\left|\xi_{k}^{t}\right|^{2} \leq \kappa_{2}\left|\xi_{k}^{t_{i}}\right|^{2}$ for all $t \in\left[t_{i}, t_{i+1}\right)$ and

$$
\kappa_{2}=\frac{c_{2}\left(c_{1} c_{5}+c_{2} c_{4}\right)}{c_{1}^{2} c_{5}-c_{1} c_{2}\left(c_{4}-c_{5}\right)\left(1-e^{-\gamma}\right)},
$$

which clearly is also valid for the case $c_{5} \geq c_{4}$, i.e. $\lambda \leq 0$.

Thus, during each interval between impulses, the trajectories of the system are bounded by a constant value as in (10), and due to the convergence property given by (9), according to Definition 1, the $2 D$ system described by (7)-(8) is $E D \xi_{k}^{t}-S$.

Proof of Corollary 1: Let us calculate the divergence operator for the quadratic vector Lyapunov function $V\left(z_{k}^{t}, z_{k+1}^{t_{i+1}}\right)$ given by (18), i.e.

$$
\begin{aligned}
\operatorname{div} V\left(z_{k}^{t}, z_{k+1}^{t_{i+1}}\right)= & \frac{d V_{1}\left(z_{k}^{t}\right)}{d t}+V_{2}\left(z_{k+1}^{t_{i+1}}\right)-V_{2}\left(z_{k}^{t_{i+1}}\right) \\
= & \left(\xi_{k}^{t}\right)^{T}\left(P_{1}\left(\tau_{k}^{t}\right) A_{\xi}+A_{\xi}^{T} P_{1}\left(\tau_{k}^{t}\right)+\frac{d P_{1}\left(\tau_{k}^{t}\right)}{d t}\right) \xi_{k}^{t}+\left(\xi_{k}^{t}\right)^{T} P_{1}\left(\tau_{k}^{t}\right) D_{\xi} f+f^{T} D_{\xi}^{T} P_{1}\left(\tau_{k}^{t}\right) \xi_{k}^{t} \\
& \quad+\left(\xi_{k}^{t_{i+1}}\right)^{T}\left(I_{\xi}^{T} P_{2}(0) I_{\xi}-P_{2}\left(T_{i}\right)\right) \xi_{k}^{t} .
\end{aligned}
$$

From the $\Lambda$-inequality (see, for instance, [25]), it follows that

$$
X Y^{T}+Y X^{T} \leq X \Lambda^{-1} X^{T}+Y \Lambda Y^{T},
$$

Therefore, the LMI (23) is obtained when all the elements of $\widehat{\Xi}_{3}(\Theta)$ are merged, and it is concluded that if the set of LMIs (23) and (25) is feasible then (42) holds, i.e. $\Upsilon_{1}(\Theta) \leq 0$. that holds for every $X \in \mathbb{R}^{n \times k}, Y \in \mathbb{R}^{n \times k}$, and $0<\Lambda=\Lambda^{T} \in \mathbb{R}^{k \times k}$. Applied with $X=\left(\xi_{k}^{t}\right)^{T} P_{1}\left(\tau_{k}^{t}\right)$ and $Y=f^{T} D_{\xi}^{T}$, it follows that

$$
\left(\xi_{k}^{t}\right)^{T} P_{1}\left(\tau_{k}^{t}\right) D_{\xi} f+f^{T} D_{\xi}^{T} P_{1}\left(\tau_{k}^{t}\right) \xi_{k}^{t} \leq\left(\xi_{k}^{t}\right)^{T} P_{1}\left(\tau_{k}^{t}\right) \Lambda^{-1} P_{1}\left(\tau_{k}^{t}\right) \xi_{k}^{t}+f^{T} D_{\xi}^{T} \Lambda D_{\xi} f
$$

for any $0<\Lambda=\Lambda^{T} \in \mathbb{R}^{4 n \times 4 n}$. Therefore, the divergence can be upper bounded as

$$
\begin{aligned}
\operatorname{div} V\left(z_{k}^{t}, z_{k+1}^{t_{i+1}}\right) \leq\left(\xi_{k}^{t}\right)^{T} & \left(P_{1}\left(\tau_{k}^{t}\right) A_{\xi}+A_{\xi}^{T} P_{1}\left(\tau_{k}^{t}\right)+\frac{d P_{1}\left(\tau_{k}^{t}\right)}{d t}+P_{1}\left(\tau_{k}^{t}\right) \Lambda^{-1} P_{1}\left(\tau_{k}^{t}\right)\right) \xi_{k}^{t} \\
& +f^{T} D_{\xi}^{T} \Lambda D_{\xi} f+\left(\xi_{k}^{t_{i+1}}\right)^{T}\left(I_{\xi}^{T} P_{2}(0) I_{\xi}-P_{2}\left(T_{i}\right)\right) \xi_{k}^{t}
\end{aligned}
$$

and since $f^{T} f \leq f_{0} x^{T} x \leq f_{0}\left(\xi_{k}^{t}\right)^{T} \xi_{k}^{t}$ and $D_{\xi}^{T} D_{\xi}=2 I_{n} \rightarrow\left|D_{\xi}^{T} D_{\xi}\right| \leq 2$, it is obtained that

$$
\begin{gathered}
\operatorname{div} V\left(z_{k}^{t}, z_{k+1}^{t_{i+1}}\right) \leq\left(\xi_{k}^{t}\right)^{T}\left(P_{1}\left(\tau_{k}^{t}\right) A_{\xi}+\right. \\
\left.A_{\xi}^{T} P_{1}\left(\tau_{k}^{t}\right)+\frac{d P_{1}\left(\tau_{k}^{t}\right)}{d t}+P_{1}\left(\tau_{k}^{t}\right) \Lambda^{-1} P_{1}\left(\tau_{k}^{t}\right)+2 f_{0} \Lambda\right) \xi_{k}^{t} \\
+\left(\xi_{k}^{t_{i+1}}\right)^{T}\left(I_{\xi}^{T} P_{2}(0) I_{\xi}-P_{2}\left(T_{i}\right)\right) \xi_{k}^{t} .
\end{gathered}
$$


By Theorem 1, it follows that the divergence must satisfy (15). Thus, it is given that

$$
\begin{gathered}
\left(\begin{array}{c}
\xi_{k}^{t} \\
\xi_{k}^{t_{i+1}}
\end{array}\right)^{T}\left(\begin{array}{cc}
P_{1}\left(\tau_{k}^{t}\right) A_{\xi}+A_{\xi}^{T} P_{1}\left(\tau_{k}^{t}\right)+\frac{d P_{1}\left(\tau_{k}^{t}\right)}{d t} & \mathbf{0} \\
+P_{1}\left(\tau_{k}^{t}\right) \Lambda^{-1} P_{1}\left(\tau_{k}^{t}\right)+2 f_{0} \Lambda & I_{\xi}^{T} P_{2}(0) I_{\xi}-P_{2}\left(T_{i}\right)
\end{array}\right)\left(\begin{array}{c}
\xi_{k}^{t} \\
\xi_{k}^{t i+1}
\end{array}\right) \leq-c_{5}\left(\left|\xi_{k}^{t}\right|^{2}+\left|\xi_{k}^{t_{i+1}}\right|^{2}\right), \\
\left(\begin{array}{c}
\xi_{k}^{t} \\
\xi_{k}^{t i+1}
\end{array}\right)^{T}\left(\begin{array}{cc}
P_{1}\left(\tau_{k}^{t}\right) A_{\xi}+A_{\xi}^{T} P_{1}\left(\tau_{k}^{t}\right)+\frac{d P_{1}\left(\tau_{k}^{t}\right)}{d t} \\
+P_{1}\left(\tau_{k}^{t}\right) \Lambda^{-1} P_{1}\left(\tau_{k}^{t}\right)+2 f_{0} \Lambda+c_{5} I_{4 n} & \mathbf{0} \\
\mathbf{0} & I_{\xi}^{T} P_{2}(0) I_{\xi}-P_{2}\left(T_{i}\right)+c_{5} I_{4 n}
\end{array}\right)\left(\begin{array}{c}
\xi_{k}^{t} \\
\xi_{k}^{t i+1}
\end{array}\right) \leq 0 .
\end{gathered}
$$

By Schur's complement to the previous inequality, one gets the matrix inequality (21). Then, if (21) is feasible for all $\tau_{k}^{t} \in\left[0, T_{i}\right], i=k \in \mathbb{N}$, some $P_{1}\left(\tau_{k}^{t}\right)=P_{1}^{T}\left(\tau_{k}^{t}\right)>0$, continuously differentiable on $t$ and bounded, $P_{2}(0)=$ $P_{2}^{T}(0)>0$ and $P_{2}\left(T_{i}\right)=P_{2}^{T}\left(T_{i}\right)>0$ satisfying (19)-(20), $\Lambda=\Lambda>0$ and a constant $c_{5} \in \mathbb{R}_{\geq 0}$, the divergence will satisfy (15).

Thus, based on Theorem 1, if the constraints (16)-(17) are satisfied for the given $c_{1}, c_{2}, c_{3}, c_{4}$ and $c_{5}$; then the system (7)-(8) will be $E D \xi_{k}^{t}-S$ for any sequence $\left\{T_{i}\right\}_{i \in \mathbb{N}}$ such that $T_{i} \in\left[\frac{c_{2}}{c_{5}} \gamma, \frac{c_{2}}{c_{5}} \alpha\right]$.

Proof of Corollary 2: It is straightforward from the proof of Corollary 1.

Proof of Proposition 1: Due to the linear structure on $\tau_{k}^{t}$ given for $P_{1}$ and $P_{2}$, the matrix inequality (21) is affine in $\tau_{k}^{t}$ and its negative definiteness is given by the negativeness over the finite set $\tau_{k}^{t} \in\left\{0, T_{i}\right\}$.

Therefore, taking into account the structure given by $A_{\xi}$ and $D_{\xi}$, and the fact that $P_{11}=\operatorname{diag}\left(P_{11}^{-(1)}, P_{11}^{-(2)}, P_{11}^{-(3)}, P_{11}^{-(3)}\right)$, $P_{12}=\operatorname{diag}\left(P_{12}^{-(1)}, P_{12}^{-(2)}, \delta I_{n}, \delta I_{n}\right)$, with $\delta>0$, after some algebraic manipulations on matrix inequality $(21)$ given by Corollary 1 , it is possible to obtain the following inequalities

$$
\begin{aligned}
& \Upsilon_{1}(\Theta)=\left(\begin{array}{c|cc}
\Omega_{1}(\Theta) & \mathbf{0} & P_{11} \\
\star & \phi(\Theta) & \mathbf{0} \\
\star & \star & -\Lambda
\end{array}\right) \leq 0, \\
& \Upsilon_{2}(\Theta)=\left(\begin{array}{c|cc}
\Omega_{2}(\Theta) & \mathbf{0} & P_{12} \\
\hline \star & \phi(\Theta) & \mathbf{0} \\
\star & \star & -\Lambda
\end{array}\right) \leq 0,
\end{aligned}
$$

where $\phi(\Theta)=I_{\xi}^{T} P_{21} I_{\xi}-P_{21}-\Theta P_{22}+Q_{5}, \Lambda=\operatorname{diag}\left(I_{n}, I_{n}, \Lambda_{3}, \Lambda_{4}\right)$, and

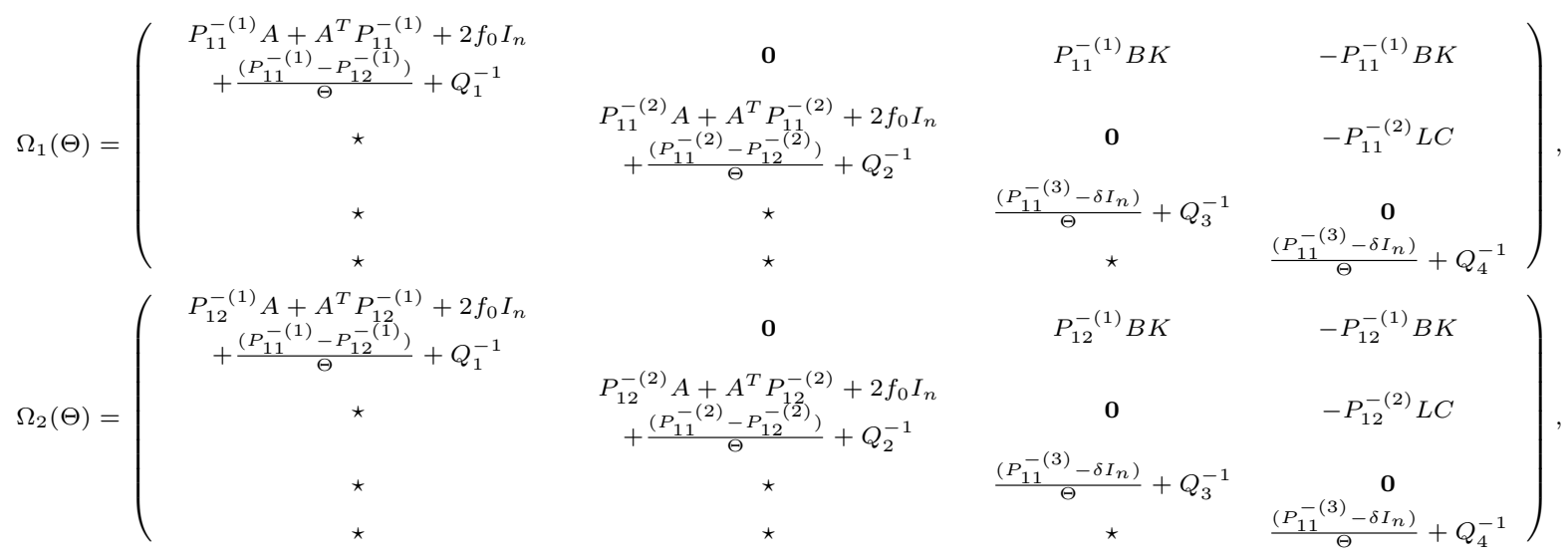

that should be satisfied for the finite set $\Theta \in\left\{T_{\min }, T_{\max }\right\}$. Let us begin with the inequality (42). Applying the quadratic non-singular transformation

$$
\mathcal{T}_{1}=\operatorname{diag}\left(P_{11}^{(1)}, P_{11}^{(2)}, I_{n}, I_{n}, I_{4 n}, I_{4 n}\right),
$$


to $(42)$, one gets

$$
W_{1}(\Theta)=\mathcal{T}_{1} \Upsilon_{1}(\Theta) \mathcal{T}_{1}^{T}=\left(\begin{array}{c|cc}
\bar{\Omega}_{1}(\Theta) & \mathbf{0} & \bar{P}_{11} \\
\hline \star & \phi(\Theta) & \mathbf{0} \\
\star & \star & -\Lambda
\end{array}\right),
$$

where

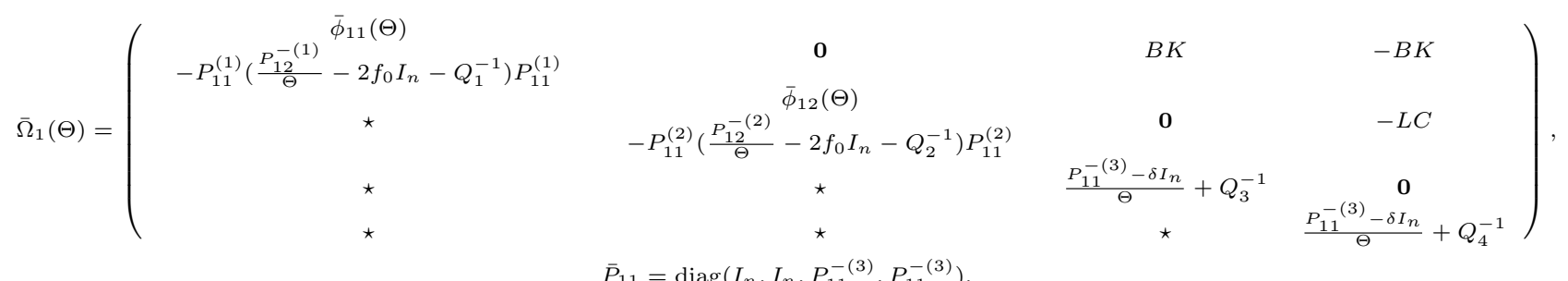

where $\bar{\phi}_{11}(\Theta)=A P_{11}^{(1)}+P_{11}^{(1)} A^{T}+P_{11}^{(1)} / \Theta$ and $\bar{\phi}_{12}(\Theta)=A P_{11}^{(2)}+P_{11}^{(2)} A^{T}+P_{11}^{(2)} / \Theta$. From $\Lambda$-inequality, applied with $Y=I$, it follows that

$$
X+X^{T} \leq X \Lambda X^{T}+\Lambda^{-1},
$$

which for $\Lambda^{-1}=\Theta P_{12}^{(1)}$ and $\Lambda^{-1}=\Theta P_{12}^{(2)}$ implies that $-P_{11}^{(1)} P_{12}^{-(1)} P_{11}^{(1)} / \Theta \leq-2 P_{11}^{(1)}+\Theta P_{12}^{(1)}$ and $-P_{11}^{(2)} P_{12}^{-(2)} P_{11}^{(2)} / \Theta \leq$ $-2 P_{11}^{(2)}+\Theta P_{12}^{(2)}$, respectively. Therefore, the matrix $W_{1}(\Theta)$ in $(44)$ can be upper estimated as $W_{1}(\Theta) \leq \bar{W}_{1}(\Theta)$, where $\bar{W}_{1}(\Theta)$ is defined as

$$
\bar{W}_{1}(\Theta)=\left(\begin{array}{c|cc}
\tilde{\Omega}_{1}(\Theta) & \mathbf{0} & \bar{P}_{11} \\
\hline \star & \phi(\Theta) & \mathbf{0} \\
\star & \star & -\Lambda
\end{array}\right),
$$

and

$$
\tilde{\Omega}_{1}(\Theta)=\left(\begin{array}{cccc}
\phi_{11}(\Theta)+P_{11}^{(1)}\left(2 f_{0} I_{n}+Q_{1}^{-1}\right) P_{11}^{(1)} & \mathbf{0} & B K & -B K \\
\star & \phi_{12}(\Theta)+P_{11}^{(2)}\left(2 f_{0} I_{n}+Q_{2}^{-1}\right) P_{11}^{(2)} & \mathbf{0} & -L C \\
\star & \star & \frac{P_{11}^{-(3)}-\delta I_{n}}{\Theta}+Q_{3}^{-1} & \mathbf{0} \\
\star & \star & \star & \frac{P_{11}^{-(3)}-\delta I_{n}}{\Theta}+Q_{4}^{-1}
\end{array}\right) \text {, }
$$

with $\phi_{11}(\Theta)=\bar{\phi}_{11}(\Theta)-2 P_{11}^{(1)}+\Theta P_{12}^{(1)}$ and $\phi_{12}(\Theta)=\bar{\phi}_{12}(\Theta)-2 P_{11}^{(2)}+\Theta P_{12}^{(2)}$. Then, it is clear that

$$
\bar{W}_{1}(\Theta)=\widetilde{W}_{1}(\Theta)+\bar{P}^{(1)}\left(2 f_{0} I_{n}+Q_{1}^{-1}\right)\left(\bar{P}^{(1)}\right)^{T}+\bar{P}^{(2)}\left(2 f_{0} I_{n}+Q_{2}^{-1}\right)\left(\bar{P}^{(2)}\right)^{T},
$$

where $\left(\bar{P}^{(1)}\right)^{T}=\left(P_{11}^{(1)}, \mathbf{0}, \ldots, \mathbf{0}\right),\left(\bar{P}^{(2)}\right)^{T}=\left(\mathbf{0}, P_{11}^{(2)}, \mathbf{0}, \ldots, \mathbf{0}\right)$ and $\widetilde{W}_{1}(\Theta)$ is given by as

$$
\widetilde{W}_{1}(\Theta)=\left(\begin{array}{c|cc}
\hat{\Omega}_{1}(\Theta) & \mathbf{0} & \bar{P}_{11} \\
\hline \star & \phi(\Theta) & \mathbf{0} \\
\star & \star & -\Lambda
\end{array}\right),
$$

where

$$
\hat{\Omega}_{1}(\Theta)=\left(\begin{array}{cccc}
\phi_{11}(\Theta) & \mathbf{0} & B K & -B K \\
\star & \phi_{12}(\Theta) & \mathbf{0} & -L C \\
\star & \star & \frac{P_{11}^{-(3)}-\delta I_{n}}{\Theta}+Q_{3}^{-1} & \mathbf{0} \\
\star & \star & \star & \frac{P_{11}^{-(1)}-\delta I_{n}}{\Theta}+Q_{4}^{-1}
\end{array}\right) .
$$

For the aperiodic case the gains were $K=(-0.3107,-0.6213), L=(2.5714,1.9137)^{T}$ corresponding to $T_{i}=0.3$.

By Schur's complement to $(46)$, it is obtained that $\bar{W}_{1}(\Theta) \leq 0$ is equivalent to $W_{2}(\Theta) \leq 0$, where $W_{2}(\Theta)$ is 
defined by

$$
W_{2}(\Theta)=\left(\begin{array}{c|cc|cc}
\widetilde{W}_{1}(\Theta) & \bar{P}^{(1)} & \bar{P}^{(1)} & \bar{P}^{(2)} & \bar{P}^{(2)} \\
\hline \star & -Q_{1} & \mathbf{0} & \mathbf{0} & \mathbf{0} \\
\star & \star & -\frac{I_{n}}{2 f_{0}} & \mathbf{0} & \mathbf{0} \\
\hline \star & \star & \star & -Q_{2} & \mathbf{0} \\
\star & \star & \star & \star & -\frac{I_{n}}{2 f_{0}}
\end{array}\right)
$$

In an analogous manner, applying the equivalent transformation

$$
\mathcal{T}_{2}=\operatorname{diag}\left(I_{n}, I_{n}, P_{11}^{(3)}, P_{11}^{(3)}, I_{4 n}, I_{4 n}, I_{4 n}\right),
$$

to the matrix $W_{2}(\Theta)$, it is obtained

$$
\Xi_{1}(\Theta)=\mathcal{T}_{2} W_{2}(\Theta) \mathcal{T}_{2}^{T}=\left(\begin{array}{c|cc|cc}
\Gamma_{1}(\Theta) & \bar{P}^{(1)} & \bar{P}^{(1)} & \bar{P}^{(2)} & \bar{P}^{(2)} \\
\hline \star & -Q_{1} & \mathbf{0} & \mathbf{0} & \mathbf{0} \\
\star & \star & -\frac{I_{n}}{2 f_{0}} & \mathbf{0} & \mathbf{0} \\
\hline \star & \star & \star & -Q_{2} & \mathbf{0} \\
\star & \star & \star & \star & -\frac{I_{n}}{2 f_{0}}
\end{array}\right)
$$

where

$\Gamma_{1}(\Theta)=\left(\begin{array}{c|cc}\check{\Omega}_{1}(\Theta) & \mathbf{0} & \tilde{P}_{11} \\ \hline \star & \phi(\Theta) & \mathbf{0} \\ \star & \star & -\Lambda\end{array}\right), \check{\Omega}_{1}(\Theta)=\left(\begin{array}{cccc}\phi_{11}(\Theta) & 0 & B K P_{11}^{(3)} & -B K P_{11}^{(3)} \\ \star & \phi_{12}(\Theta) & -L C P_{11}^{(3)} \\ \star & \star & P_{11}^{(3)}\left(\frac{P_{11}^{-(3)}-\delta I_{n}}{\Theta}+Q_{3}^{-1}\right) P_{11}^{(3)} & 0 \\ \star & \star & \star & P_{11}^{(3)}\left(\frac{P_{11}^{-(3)}-\delta I_{n}}{\Theta}+Q_{4}^{-1}\right) P_{11}^{(3)}\end{array}\right)$

with $\tilde{P}_{11}=\operatorname{diag}\left(I_{n}, I_{n}, I_{n}, I_{n}\right)$. By $\Lambda$-inequality, it follows that $\left(P_{11}^{(3)}-\delta P_{11}^{(3)} I_{n} P_{11}^{(3)}\right) / \Theta \leq P_{11}^{(3)} / \Theta-2 P_{11}^{(3)}+\Theta I_{n} / \delta=$ $\phi_{3}(\Theta)$. Therefore, the matrix $\Xi_{1}(\Theta)$ is upper estimated as $\Xi_{1}(\Theta) \leq \bar{\Xi}_{1}(\Theta)$, where $\bar{\Xi}_{1}(\Theta)$ is given by

$$
\bar{\Xi}_{1}(\Theta)=\left(\begin{array}{c|cc|cc}
\bar{\Gamma}_{1}(\Theta) & \bar{P}^{(1)} & \bar{P}^{(1)} & \bar{P}^{(2)} & \bar{P}^{(2)} \\
\hline \star & -Q_{1} & \mathbf{0} & \mathbf{0} & \mathbf{0} \\
\star & \star & -\frac{I_{n}}{2 f_{0}} & \mathbf{0} & \mathbf{0} \\
\hline \star & \star & \star & -Q_{2} & \mathbf{0} \\
\star & \star & \star & \star & -\frac{I_{n}}{2 f_{0}}
\end{array}\right),
$$

where

$$
\bar{\Gamma}_{1}(\Theta)=\left(\begin{array}{c|cc}
\tilde{\Gamma}_{1}(\Theta) & \mathbf{0} & \tilde{P}_{11} \\
\hline \star & \phi(\Theta) & \mathbf{0} \\
\star & \star & -\Lambda
\end{array}\right), \tilde{\Gamma}_{1}(\Theta)=\left(\begin{array}{cccc}
\phi_{11}(\Theta) & 0 & B K P_{11}^{(3)} & -B K P_{11}^{(3)} \\
\star & \phi_{12}(\Theta) & 0 \\
\star & \star & \phi_{13}(\Theta)+P_{11}^{(3)} Q_{3}^{-1} P_{11}^{(3)} & -L C P_{11}^{(3)} \\
\star & \star & \star & 0 \\
& & \star & \phi_{3}(\Theta)+P_{11}^{(3)} Q_{4}^{-1} P_{11}^{(3)}
\end{array}\right) .
$$

Then, it is given that

$$
\bar{\Xi}_{1}(\Theta)=\widetilde{\Xi}_{1}(\Theta)+\bar{P}^{(3)} Q_{3}^{-1}\left(\bar{P}^{(3)}\right)^{T}+\bar{P}^{(4)} Q_{4}^{-1}\left(\bar{P}^{(4)}\right)^{T},
$$

where $\left(\bar{P}^{(3)}\right)^{T}=\left(\mathbf{0}, \mathbf{0}, P_{11}^{(3)}, \mathbf{0}, \ldots, \mathbf{0}\right),\left(\bar{P}^{(4)}\right)^{T}=\left(\mathbf{0}, \mathbf{0}, \mathbf{0}, P_{11}^{(3)}, \mathbf{0}, \ldots, \mathbf{0}\right)$ and $\widetilde{\Xi}_{1}(\Theta)$ defined by

$$
\widetilde{\Xi}_{1}(\Theta)=\left(\begin{array}{c|cc|cc}
\Pi_{1}(\Theta) & \bar{P}^{(1)} & \bar{P}^{(1)} & \bar{P}^{(2)} & \bar{P}^{(2)} \\
\hline \star & -Q_{1} & \mathbf{0} & \mathbf{0} & \mathbf{0} \\
\star & \star & -\frac{I_{n}}{2 f_{0}} & \mathbf{0} & \mathbf{0} \\
\hline \star & \star & \star & -Q_{2} & \mathbf{0} \\
\star & \star & \star & \star & -\frac{I_{n}}{2 f_{0}}
\end{array}\right),
$$


where

$$
\Pi_{1}(\Theta)=\left(\begin{array}{c|cc}
\tilde{\Pi}_{1}(\Theta) & \mathbf{0} & \tilde{P}_{11} \\
\hline \star & \phi(\Theta) & 0 \\
\star & \star & -\Lambda
\end{array}\right), \tilde{\Pi}_{1}(\Theta)=\left(\begin{array}{cccc}
\phi_{11}(\Theta) & \mathbf{0} & B Y_{K} & -B Y_{K} \\
\star & \phi_{12}(\Theta) & 0 & -L C P_{11}^{(3)} \\
\star & \star & \phi_{3}(\Theta) & 0 \\
\star & \star & \star & \phi_{3}(\Theta)
\end{array}\right),
$$

with $Y_{K}=K P_{11}^{(3)}$. Then, applying Schur's complement to $(51)$, it is obtained that $\bar{\Xi}_{1}(\Theta) \leq 0$ is equivalent to $\Xi_{2}(\Theta) \leq 0$, where $\Xi_{2}(\Theta)$ is defined as

$$
\Xi_{2}(\Theta)=\left(\begin{array}{c|c|c}
\widetilde{\Xi}_{1}(\Theta) & \bar{P}^{(3)} & \bar{P}^{(4)} \\
\hline \star & -Q_{3} & \mathbf{0} \\
\hline \star & \star & -Q_{4}
\end{array}\right)
$$

Then, the bilinear term $-L C P_{11}^{(3)}$ is simplified as follows. By $\Lambda$-inequality with

$$
X^{T}=\left(\begin{array}{lllllll}
\mathbf{0} & \mathbf{0} & \mathbf{0} & P_{11}^{(3)} & \mathbf{0} & \cdots & \mathbf{0}
\end{array}\right)^{T}, Y=\left(\begin{array}{lllll}
\mathbf{0} & -L C & \mathbf{0} & \cdots & \mathbf{0}
\end{array}\right),
$$

the matrix $\Xi_{2}(\Theta)$ can be upper estimated as $\Xi_{2}(\Theta) \leq \bar{\Xi}_{2}(\Theta)$, where $\bar{\Xi}_{2}(\Theta)$ is defined by

$$
\bar{\Xi}_{2}(\Theta)=\left(\begin{array}{c|c|c}
\Psi_{1}(\Theta) & \bar{P}^{(3)} & \bar{P}^{(4)} \\
\hline \star & -Q_{3} & \mathbf{0} \\
\hline \star & \star & -Q_{4}
\end{array}\right)
$$

where

$$
\Psi_{1}(\Theta)=\left(\begin{array}{c|cc|cc}
\bar{\Psi}_{1}(\Theta) & \bar{P}^{(1)} & \bar{P}^{(1)} & \bar{P}^{(2)} & \bar{P}^{(2)} \\
\hline \star & -Q_{1} & \mathbf{0} & \mathbf{0} & \mathbf{0} \\
\star & \star & -\frac{I_{n}}{2 f_{0}} & \mathbf{0} & \mathbf{0} \\
\hline \star & \star & \star & -Q_{2} & \mathbf{0} \\
\star & \star & \star & \star & -\frac{I_{n}}{2 f_{0}}
\end{array}\right), \bar{\Psi}_{1}(\Theta)=\left(\begin{array}{c|cc}
\Phi_{1}(\Theta) & \mathbf{0} & \tilde{P}_{11} \\
\hline \star & \phi(\Theta) & \mathbf{0} \\
\star & \star & -\Lambda
\end{array}\right),
$$

and

$$
\Phi_{1}(\Theta)=\left(\begin{array}{cccc}
\phi_{11}(\Theta) & \mathbf{0} & B Y_{K} & -B Y_{K} \\
\star & \bar{\phi}_{12}(\Theta) & \mathbf{0} & \mathbf{0} \\
\star & \star & \phi_{3}(\Theta) & \mathbf{0} \\
\star & \star & \star & \bar{\phi}_{3}(\Theta)
\end{array}\right)
$$

with

$$
\begin{aligned}
& \bar{\phi}_{12}(\Theta)=\phi_{12}(\Theta)+L C \bar{\Lambda}_{1} C^{T} L^{T}, \\
& \bar{\phi}_{3}(\Theta)=\phi_{3}(\Theta)+P_{11}^{(3)} \bar{\Lambda}_{1}^{-1} P_{11}^{(3)},
\end{aligned}
$$

for any $\bar{\Lambda}_{1}=\bar{\Lambda}_{1}^{T}>0$. Let $R_{1}, R_{2}>0$ be new matrix variables. Then, applying $\Lambda$-inequality to $\bar{\phi}_{12}$ it follows that

$$
\begin{gathered}
\phi_{12}(\Theta)+L C \bar{\Lambda}_{1} C^{T} L^{T} \leq \phi_{12}(\Theta)-L C-C^{T} L^{T}+\bar{\Lambda}_{1}^{-1} \leq-R_{1}, \\
\phi_{3}(\Theta)+P_{11}^{(3)} \bar{\Lambda}_{1}^{-1} P_{11}^{(3)} \leq-R_{2},
\end{gathered}
$$

and applying Schur's complement one gets the LMIs (25), i.e.

$$
\left(\begin{array}{cc}
R_{1}+\phi_{12}(\Theta)-L C-C^{T} L^{T} & I_{n} \\
I_{n} & -\bar{\Lambda}_{1}
\end{array}\right) \leq 0,\left(\begin{array}{cc}
R_{2}+\phi_{3}(\Theta) & P_{11}^{(3)} \\
P_{11}^{(3)} & -\bar{\Lambda}_{1}
\end{array}\right) \leq 0 .
$$

Thus the term $\Phi_{1}$ is upper estimated as $\Phi_{1}(\Theta) \leq \bar{\Phi}_{1}(\Theta)$, where $\bar{\Phi}_{1}(\Theta)$ is defined by

$$
\bar{\Phi}_{1}(\Theta)=\left(\begin{array}{cccc}
\phi_{11}(\Theta) & \mathbf{0} & B Y_{K} & -B Y_{K} \\
\star & -R_{1} & \mathbf{0} & \mathbf{0} \\
\star & \star & \phi_{3}(\Theta) & \mathbf{0} \\
\star & \star & \star & -R_{2}
\end{array}\right)
$$


Therefore, the matrix $\bar{\Xi}_{2}(\Theta)$ can be upper estimated as $\bar{\Xi}_{2}(\Theta) \leq \Xi_{3}(\Theta)$, where $\Xi_{3}(\Theta)$, is defined by

$$
\Xi_{3}(\Theta)=\left(\begin{array}{c|c|c}
\widehat{\Psi}_{1}(\Theta) & \bar{P}^{(3)} & \bar{P}^{(4)} \\
\hline \star & -Q_{3} & \mathbf{0} \\
\hline \star & \star & -Q_{4}
\end{array}\right),
$$

where

$$
\widehat{\Psi}_{1}(\Theta)=\left(\begin{array}{c|cc|cc}
\grave{\Psi}_{1}(\Theta) & \bar{P}^{(1)} & \bar{P}^{(1)} & \bar{P}^{(2)} & \bar{P}^{(2)} \\
\hline \star & -Q_{1} & \mathbf{0} & \mathbf{0} & \mathbf{0} \\
\star & \star & -\frac{I_{n}}{2 f_{0}} & \mathbf{0} & \mathbf{0} \\
\hline \star & \star & \star & -Q_{2} & \mathbf{0} \\
\star & \star & \star & \star & -\frac{I_{n}}{2 f_{0}}
\end{array}\right), \grave{\Psi}_{1}(\Theta)=\left(\begin{array}{cc|cc}
\bar{\Phi}_{1}(\Theta) & \mathbf{0} & \tilde{P}_{11} \\
\hline \star & \phi(\Theta) & \mathbf{0} \\
\star & \star & -\Lambda
\end{array}\right) .
$$

Therefore, the LMI (23) is obtained when all the elements of $\Xi_{3}(\Theta)$ are merged, and it is concluded that if the set of LMIs (23) and (25) is feasible then (42) holds, i.e. $\Upsilon_{1}(\Theta) \leq 0$.

To conclude the proof, it is clear that a similar method may be used to obtain the LMIs (24) and (26) by means of inequality (43), and prove that if the set of LMIs (24) and (26) is feasible then (42) also holds, i.e. $\Upsilon_{2}(\Theta) \leq 0$. This procedure is omitted for the sake of brevity. Thus, the theorem is proven.

Proof of Proposition 2: Due to the linear structure on $\tau_{k}^{t}$ given for $P_{1}$ and $P_{2}$, the matrix inequality (21) is affine in $\tau_{k}^{t}$ and its negative definiteness is given by the negativeness over the finite set $\tau_{k}^{t} \in\left\{0, T_{i}\right\}$.

Thus, given the structure of $A_{\xi}$ and $D_{\xi}$, and the fact that $P_{11}=\operatorname{diag}\left(P_{11}^{-(1)}, P_{11}^{-(2)}, P_{11}^{-(3)}, P_{11}^{-(3)}\right), P_{12}=$ $\operatorname{diag}\left(P_{12}^{-(1)}, P_{12}^{-(2)}, \delta I_{n}, \delta I_{n}\right)$, with $\delta>0$, after some algebraic manipulations on matrix inequality (22) given by Corollary 2 , it is possible to obtain the following inequality

$$
\begin{aligned}
& \Upsilon_{1}(\Theta)=\left(\begin{array}{c|c}
\Omega_{1}(\Theta) & \mathbf{0} \\
\hline \mathbf{0} & \phi(\Theta)
\end{array}\right) \leq 0, \\
& \Upsilon_{2}(\Theta)=\left(\begin{array}{c|c}
\Omega_{2}(\Theta) & \mathbf{0} \\
\hline \mathbf{0} & \phi(\Theta)
\end{array}\right) \leq 0
\end{aligned}
$$

where $\phi(\Theta)=I_{\xi}^{T} P_{21} I_{\xi}-P_{21}-\Theta P_{22}+Q_{5}$ and

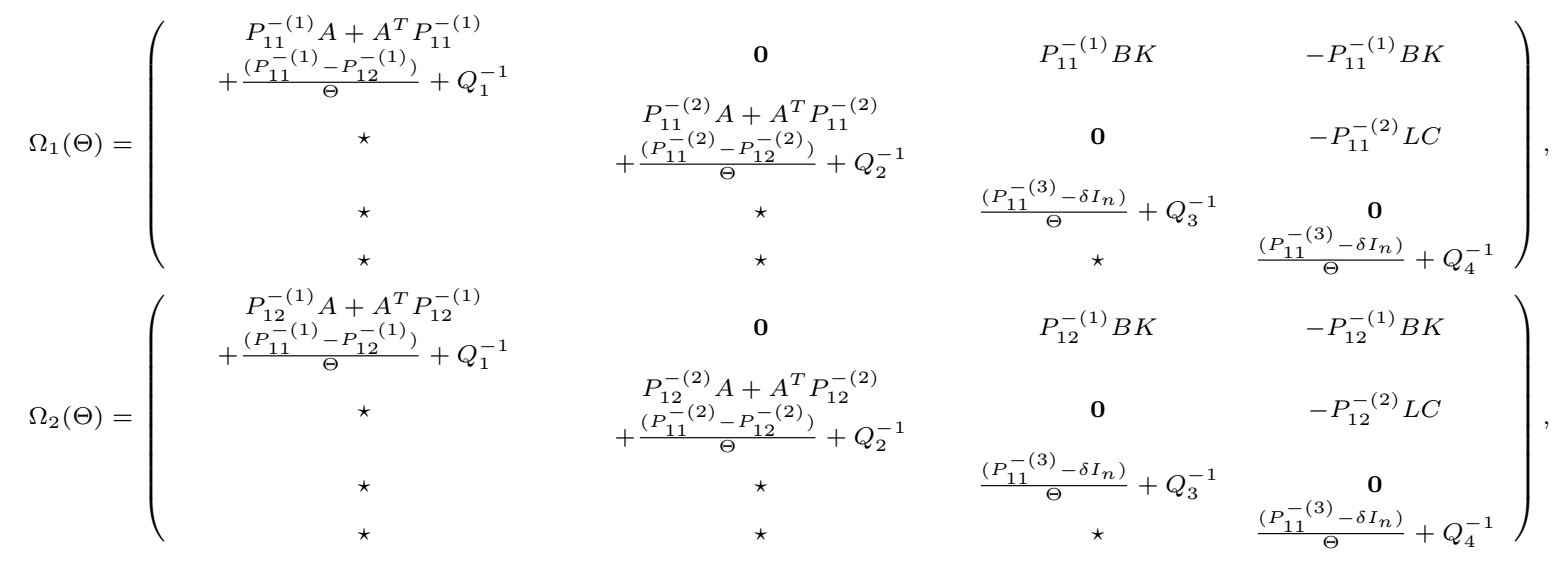

that should hold for the finite set $\Theta \in\left\{T_{\min }, T_{\max }\right\}$. Let us begin with the inequality (56). Applying the quadratic non-singular transformation

$$
\mathcal{T}_{1}=\operatorname{diag}\left(P_{11}^{(1)}, P_{11}^{(2)}, I_{n}, I_{n}, I_{4 n}\right),
$$

to $(56)$, one gets

$$
W_{1}(\Theta)=\mathcal{T}_{1} \Upsilon_{1}(\Theta) \mathcal{T}_{1}^{T}=\left(\begin{array}{c|c}
\bar{\Omega}_{1}(\Theta) & \mathbf{0} \\
\hline \mathbf{0} & \phi(\Theta)
\end{array}\right)
$$

where 
$\bar{\Omega}_{1}(\Theta)=\left(\begin{array}{cccc}\bar{\phi}_{11}(\Theta)-P_{11}^{(1)}\left(\frac{P_{12}^{-(1)}}{\Theta}-Q_{1}^{-1}\right) P_{11}^{(1)} & \mathbf{0} & B K & -B K \\ \star & \bar{\phi}_{12}(\Theta)-P_{11}^{(2)}\left(\frac{P_{12}^{-(2)}}{\Theta}-Q_{2}^{-1}\right) P_{11}^{(2)} & \mathbf{0} & -L C \\ \star & \star & \frac{P_{11}^{-(3)}-\delta I_{n}}{\Theta}+Q_{3}^{-1} & \mathbf{0} \\ \star & \star & \star & \frac{P_{11}^{-(3)}-\delta I_{n}}{\Theta}+Q_{4}^{-1}\end{array}\right)$,

where $\bar{\phi}_{11}(\Theta)=A P_{11}^{(1)}+P_{11}^{(1)} A^{T}+P_{11}^{(1)} / \Theta$ and $\bar{\phi}_{12}(\Theta)=A P_{11}^{(2)}+P_{11}^{(2)} A^{T}+P_{11}^{(2)} / \Theta$. From $\Lambda$-inequality, it follows that $-P_{11}^{(1)} P_{12}^{-(1)} P_{11}^{(1)} / \Theta \leq-2 P_{11}^{(1)}+\Theta P_{12}^{(1)}$ and $-P_{11}^{(2)} P_{12}^{-(2)} P_{11}^{(2)} / \Theta \leq-2 P_{11}^{(2)}+\Theta P_{12}^{(2)}$, respectively. Therefore, the matrix $W_{1}(\Theta)$ in $(58)$ can be upper estimated as $W_{1}(\Theta) \leq \bar{W}_{1}(\Theta)$, where $\bar{W}_{1}(\Theta)$ is defined as

$$
\bar{W}_{1}(\Theta)=\left(\begin{array}{c|c}
\tilde{\Omega}_{1}(\Theta) & \mathbf{0} \\
\hline \mathbf{0} & \phi_{1}(\Theta)
\end{array}\right),
$$

and

$$
\tilde{\Omega}_{1}(\Theta)=\left(\begin{array}{cccc}
\phi_{11}(\Theta)+P_{11}^{(1)} Q_{1}^{-1} P_{11}^{(1)} & \mathbf{0} & B K & -B K \\
\star & \phi_{12}(\Theta)+P_{11}^{(2)} Q_{2}^{-1} P_{11}^{(2)} & \mathbf{0} & -L C \\
\star & \star & \frac{P_{11}^{-(3)}-\delta I_{n}}{\Theta}+Q_{3}^{-1} & \mathbf{0} \\
\star & \star & \star & \frac{P_{11}^{-(3)}-\delta I_{n}}{\Theta}+Q_{4}^{-1}
\end{array}\right),
$$

with $\phi_{11}(\Theta)=\bar{\phi}_{11}(\Theta)-2 P_{11}^{(1)}+\Theta P_{12}^{(1)}$ and $\phi_{12}(\Theta)=\bar{\phi}_{12}(\Theta)-2 P_{11}^{(2)}+\Theta P_{12}^{(2)}$. Then, it is clear that

$$
\bar{W}_{1}(\Theta)=\widetilde{W}_{1}(\Theta)+\bar{P}^{(1)} Q_{1}^{-1}\left(\bar{P}^{(1)}\right)^{T}+\bar{P}^{(2)} Q_{2}^{-1}\left(\bar{P}^{(2)}\right)^{T},
$$

where $\left(\bar{P}^{(1)}\right)^{T}=\left(P_{11}^{(1)}, \mathbf{0}, \ldots, \mathbf{0}\right),\left(\bar{P}^{(2)}\right)^{T}=\left(\mathbf{0}, P_{11}^{(2)}, \mathbf{0}, \ldots, \mathbf{0}\right)$ and $\widetilde{W}_{1}(\Theta)$ is given by as

$$
\widetilde{W}_{1}(\Theta)=\left(\begin{array}{c|c}
\hat{\Omega}_{1}(\Theta) & \mathbf{0} \\
\hline \mathbf{0} & \phi_{1}(\Theta)
\end{array}\right),
$$

where

$$
\hat{\Omega}_{1}(\Theta)=\left(\begin{array}{cccc}
\phi_{11}(\Theta) & \mathbf{0} & B K & -B K \\
\star & \phi_{12}(\Theta) & \mathbf{0} & -L C \\
\star & \star & \frac{P_{11}^{-(3)}-\delta I_{n}}{\Theta}+Q_{3}^{-1} & \mathbf{0} \\
\star & \star & \star & \frac{P_{11}^{-(1)}-\delta I_{n}}{\Theta}+Q_{4}^{-1}
\end{array}\right)
$$

Then, by Schur's complement to (60) twice, it is obtained that $\bar{W}_{1}(\Theta) \leq 0$ is equivalent to $W_{2}(\Theta) \leq 0$, where $W_{2}(\Theta)$ is defined as

$$
W_{2}(\Theta)=\left(\begin{array}{c|c|c}
\widetilde{W}_{1}(\Theta) & \bar{P}^{(1)} & \bar{P}^{(2)} \\
\hline \star & -Q_{1} & \mathbf{0} \\
\hline \star & \star & -Q_{2}
\end{array}\right) .
$$

In a similar way, applying the equivalent transformation

$$
\mathcal{T}_{2}=\operatorname{diag}\left(I_{n}, I_{n}, P_{11}^{(3)}, P_{11}^{(3)}, I_{n}, I_{4 n}, I_{n}\right),
$$

to the matrix $W_{2}(\Theta)$, it is obtained

$$
\Xi_{1}(\Theta)=\mathcal{T}_{2} W_{2}(\Theta) \mathcal{T}_{2}^{T}=\left(\begin{array}{c|c|c}
\Gamma_{1}(\Theta) & \bar{P}^{(1)} & \bar{P}^{(2)} \\
\hline \star & -Q_{1} & \mathbf{0} \\
\hline \star & \star & -Q_{2}
\end{array}\right)
$$


where

$$
\Gamma_{1}(\Theta)=\left(\begin{array}{c|c}
\check{\Omega}_{1}(\Theta) & \mathbf{0} \\
\hline \mathbf{0} & \phi(\Theta)
\end{array}\right), \check{\Omega}_{1}(\Theta)=\left(\begin{array}{cccc}
\phi_{11}(\Theta) & \mathbf{0} & B K P_{11}^{(3)} & -B K P_{11}^{(3)} \\
\star & \phi_{12}(\Theta) & 0 & -L C P_{11}^{(3)} \\
\star & \star & P_{11}^{(3)}\left(\frac{P_{11}^{-(3)}-\delta I_{n}}{\Theta}+Q_{3}^{-1}\right) P_{11}^{(3)} & \mathbf{0} \\
\star & \star & \star & P_{11}^{(3)}\left(\frac{P_{11}^{-(3)}-\delta I_{n}}{\Theta}+Q_{4}^{-1}\right) P_{11}^{(3)}
\end{array}\right) .
$$

By $\Lambda$-inequality, it follows that $\left(P_{11}^{(3)}-\delta P_{11}^{(3)} I_{n} P_{11}^{(3)}\right) / \Theta \leq P_{11}^{(3)} / \Theta-2 P_{11}^{(3)}+\Theta I_{n} / \delta=\phi_{3}(\Theta)$. Therefore, the matrix $\Xi_{1}(\Theta)$ is upper estimated as $\Xi_{1}(\Theta) \leq \bar{\Xi}_{1}(\Theta)$, where $\bar{\Xi}_{1}(\Theta)$ is given by

$$
\Xi_{1}(\Theta)=\left(\begin{array}{c|c|c}
\bar{\Gamma}_{1}(\Theta) & \bar{P}^{(1)} & \bar{P}^{(2)} \\
\hline \star & -Q_{1} & \mathbf{0} \\
\hline \star & \star & -Q_{2}
\end{array}\right)
$$

where

$$
\bar{\Gamma}_{1}(\Theta)=\left(\begin{array}{c|c}
\tilde{\Gamma}_{1}(\Theta) & \mathbf{0} \\
\hline \mathbf{0} & \phi(\Theta)
\end{array}\right), \tilde{\Gamma}_{1}(\Theta)=\left(\begin{array}{cccc}
\phi_{11}(\Theta) & \mathbf{0} & B K P_{11}^{(3)} & -B K P_{11}^{(3)} \\
\star & \phi_{12}(\Theta) & \mathbf{0} & -L C P_{11}^{(3)} \\
\star & \star & \phi_{13}(\Theta)+P_{11}^{(3)} Q_{3}^{-1} P_{11}^{(3)} & \mathbf{0} \\
\star & \star & \star & \phi_{3}(\Theta)+P_{11}^{(3)} Q_{4}^{-1} P_{11}^{(3)}
\end{array}\right) .
$$

Then, it is given that

$$
\Xi_{1}(\Theta)=\widetilde{\Xi}_{1}(\Theta)+\bar{P}^{(3)} Q_{3}^{-1}\left(\bar{P}^{(3)}\right)^{T}+\bar{P}^{(4)} Q_{4}^{-1}\left(\bar{P}^{(4)}\right)^{T},
$$

where $\left(\bar{P}^{(3)}\right)^{T}=\left(\mathbf{0}, \mathbf{0}, P_{11}^{(3)}, \mathbf{0}, \ldots, \mathbf{0}\right),\left(\bar{P}^{(4)}\right)^{T}=\left(\mathbf{0}, \mathbf{0}, \mathbf{0}, P_{11}^{(3)}, \mathbf{0}, \ldots, \mathbf{0}\right)$ and $\widetilde{\Xi}_{1}(\Theta)$ defined by

$$
\widetilde{\Xi}_{1}(\Theta)=\left(\begin{array}{c|c|c}
\Pi_{1}(\Theta) & \bar{P}^{(1)} & \bar{P}^{(2)} \\
\hline \star & -Q_{1} & \mathbf{0} \\
\hline \star & \star & -Q_{2}
\end{array}\right)
$$

where

$$
\Pi_{1}(\Theta)=\left(\begin{array}{c|c}
\tilde{\Pi}_{1}(\Theta) & \mathbf{0} \\
\hline \mathbf{0} & \phi(\Theta)
\end{array}\right), \tilde{\Pi}_{1}(\Theta)=\left(\begin{array}{cccc}
\phi_{11}(\Theta) & \mathbf{0} & B Y_{K} & -B Y_{K} \\
\star & \phi_{12}(\Theta) & \mathbf{0} & -L C P_{11}^{(3)} \\
\star & \star & \phi_{3}(\Theta) & \mathbf{0} \\
\star & \star & \star & \phi_{3}(\Theta)
\end{array}\right),
$$

with $Y_{K}=K P_{11}^{(3)}$. Thus, applying Schur's complement to (66), it is obtained that $\Xi_{1}(\Theta) \leq 0$ is equivalent to $\Xi_{2}(\Theta) \leq 0$, where $\Xi_{2}(\Theta)$ is defined as

$$
\Xi_{2}(\Theta)=\left(\begin{array}{c|c|c}
\widetilde{\Xi}_{1}(\Theta) & \bar{P}^{(3)} & \bar{P}^{(4)} \\
\hline \star & -Q_{3} & \mathbf{0} \\
\hline \star & \star & -Q_{4}
\end{array}\right)
$$

Then, the bilinear term $-L C P_{11}^{(3)}$ is simplified as follows. By $\Lambda$-inequality with

$$
X^{T}=\left(\begin{array}{lllllll}
\mathbf{0} & \mathbf{0} & \mathbf{0} & P_{11}^{(3)} & \mathbf{0} & \cdots & \mathbf{0}
\end{array}\right)^{T}, Y=\left(\begin{array}{lllll}
\mathbf{0} & -L C & \mathbf{0} & \cdots & \mathbf{0}
\end{array}\right),
$$

the matrix $\Xi_{2}(\Theta)$ can be upper estimated as $\Xi_{2}(\Theta) \leq \bar{\Xi}_{2}(\Theta)$, where $\bar{\Xi}_{2}(\Theta)$ is defined by

$$
\bar{\Xi}_{2}(\Theta)=\left(\begin{array}{c|c|c}
\Psi_{1}(\Theta) & \bar{P}^{(3)} & \bar{P}^{(4)} \\
\hline \star & -Q_{3} & \mathbf{0} \\
\hline \star & \star & -Q_{4}
\end{array}\right),
$$

where

$$
\Psi_{1}(\Theta)=\left(\begin{array}{c|c|c}
\bar{\Psi}_{1}(\Theta) & \bar{P}^{(1)} & \bar{P}^{(2)} \\
\hline \star & -Q_{1} & \mathbf{0} \\
\hline \star & \star & -Q_{2}
\end{array}\right), \bar{\Psi}_{1}(\Theta)=\left(\begin{array}{c|c}
\Phi_{1}(\Theta) & \mathbf{0} \\
\hline \star & \phi(\Theta)
\end{array}\right),
$$

and 


$$
\Phi_{1}(\Theta)=\left(\begin{array}{cccc}
\phi_{11}(\Theta) & \mathbf{0} & B Y_{K} & -B Y_{K} \\
\star & \bar{\phi}_{12}(\Theta) & \mathbf{0} & \mathbf{0} \\
\star & \star & \phi_{3}(\Theta) & \mathbf{0} \\
\star & \star & \star & \bar{\phi}_{3}(\Theta)
\end{array}\right)
$$

with

$$
\begin{aligned}
& \bar{\phi}_{12}(\Theta)=\phi_{12}(\Theta)+L C \bar{\Lambda}_{1} C^{T} L^{T}, \\
& \bar{\phi}_{3}(\Theta)=\phi_{3}(\Theta)+P_{11}^{(3)} \bar{\Lambda}_{1}^{-1} P_{11}^{(3)},
\end{aligned}
$$

for any $\bar{\Lambda}_{1}=\bar{\Lambda}_{1}^{T}>0$. Let $R_{1}, R_{2}>0$ be new matrix variables. Then, applying $\Lambda$-inequality to $\bar{\phi}_{12}$ it follows that

$$
\begin{gathered}
\phi_{12}(\Theta)+L C \bar{\Lambda}_{1} C^{T} L^{T} \leq \phi_{12}(\Theta)-L C-C^{T} L^{T}+\bar{\Lambda}_{1}^{-1} \leq-R_{1}, \\
\phi_{3}(\Theta)+P_{11}^{(3)} \bar{\Lambda}_{1}^{-1} P_{11}^{(3)} \leq-R_{2},
\end{gathered}
$$

and applying Schur's complement one gets the LMIs (29), i.e.

$$
\left(\begin{array}{cc}
R_{1}+\phi_{12}(\Theta)-L C-C^{T} L^{T} & I_{n} \\
I_{n} & -\bar{\Lambda}_{1}
\end{array}\right) \leq 0,\left(\begin{array}{cc}
R_{2}+\phi_{3}(\Theta) & P_{11}^{(3)} \\
P_{11}^{(3)} & -\bar{\Lambda}_{1}
\end{array}\right) \leq 0 .
$$

Thus the term $\Phi_{1}$ is upper estimated as $\Phi_{1}(\Theta) \leq \bar{\Phi}_{1}(\Theta)$, where $\bar{\Phi}_{1}(\Theta)$ is defined by

$$
\bar{\Phi}_{1}(\Theta)=\left(\begin{array}{cccc}
\phi_{11}(\Theta) & \mathbf{0} & B Y_{K} & -B Y_{K} \\
\star & -R_{1} & \mathbf{0} & \mathbf{0} \\
\star & \star & \phi_{3}(\Theta) & \mathbf{0} \\
\star & \star & \star & -R_{2}
\end{array}\right)
$$

Therefore, the matrix $\bar{\Xi}_{2}(\Theta)$ can be upper estimated as $\bar{\Xi}_{2}(\Theta) \leq \Xi_{3}(\Theta)$, where $\Xi_{3}(\Theta)$, is defined by

$$
\Xi_{3}(\Theta)=\left(\begin{array}{c|c|c}
\widehat{\Psi}_{1}(\Theta) & \bar{P}^{(3)} & \bar{P}^{(4)} \\
\hline \star & -Q_{3} & \mathbf{0} \\
\hline \star & \star & -Q_{4}
\end{array}\right)
$$

where

$$
\widehat{\Psi}_{1}(\Theta)=\left(\begin{array}{c|c|c}
\grave{\Psi}_{1}(\Theta) & \bar{P}^{(1)} & \bar{P}^{(2)} \\
\hline \star & -Q_{1} & \mathbf{0} \\
\hline \star & \star & -Q_{2}
\end{array}\right), \grave{\Psi}_{1}(\Theta)=\left(\begin{array}{c|c}
\bar{\Phi}_{1}(\Theta) & \mathbf{0} \\
\hline \star & \phi(\Theta)
\end{array}\right) .
$$

Therefore, the LMI (27) is obtained when all the elements of $\widehat{\Xi}_{3}(\Theta)$ are merged, and one conclude that if the set of LMIs $(27)$ and $(29)$ is feasible then $\Upsilon_{1}(\Theta) \leq 0$.

To conclude the proof, it is clear that a similar method may be used to obtain the LMIs (28) and (30) by means of inequality $\Upsilon_{2}(\Theta) \leq 0$. However, this procedure is omitted for the sake of brevity. Hence, the theorem is proven.

\section{References}

[1] T. Ahmed-Ali, I. Karafyllis, and F. Lamnabhi-Lagarrigue. Global exponential sampled-data observers for nonlinear systems with delayed measurements. Systems \& Control Letters, 62:539-549, 2013.

[2] N.W. Bauer, M.C.F. Donkers, N. van de Wouw, and W.P.M.H. Heemels. Decentralized observer-based control via networked communication. Automatica, 49:2074-2086, 2013.

[3] C. Briat. Convex conditions for robust stability analysis and stabilization of linear aperiodic impulsive and sampled-data systems under dwell-time constraints. Automatica, 49:3449-3457, 2013.

[4] C. Briat and A. Seuret. Convex dwell-time characterizations for uncertain linear impulsive systems. IEEE Transactions on Automatic Control, 57(12):3241-3246, 2012. 
[5] C. Briat and A. Seuret. A looped-functional approach for robust stability of linear impulsive systems. Systems \& Control Letters, 61(10):980-988, 2012.

[6] D. Dačić and D. Nešić. Observer design for wired linear networked control systems using matrix inequalities. Automatica, 44(11):2840-2848, 2008.

[7] F. Deza, E. Busvelle, J. Gauthier, and D. Rakotopora. High gain estimation for nonlinear systems. Systems 83 Control Letters, 18(4):295-299, 1992.

[8] M. Farza, M. M'Saad, M. L. Fall, E. Pigeon, O. Gehan, and K. Busawon. Continuous-discrete time observers for a class of MIMO nonlinear systems. IEEE Transactions on Automatic Control, 59(4):1060-1065, 2014.

[9] F. Ferrante, F. Gouaisbaut, R.G. Sanfelice, and S. Tarbouriech. State estimation of linear systems in the presence of sporadic measurements. Automatica, 73:101-109, 2016.

[10] E. Fridman. A refined input delay approach to sampled-data control. Automatica, 46(2):421-427, 2010.

[11] E. Fridman, A. Seuret, and J.-P Richard. Robust sampled-data stabilization of linear systems: An input delay approach. Automatica, 40(8):1441-1446, 2004.

[12] E. Fridman, U. Shaked, and V. Suplin. Input/output delay approach to robust sampled-data $\mathcal{H}_{\infty}$ control. Systems $\mathscr{B}$ Control Letters, 54:271-282, 2005.

[13] H. Fujioka. A discrete-time approach to stability analysis of systems with aperiodic sample-and-hold devices. IEEE Transactions on Automatic Control, 54(10):2440-2445, 2009.

[14] H. Fujioka. Stability analysis of systems with aperiodic sample-and-hold devices. Automatica, 45(3):771-775, 2009.

[15] R. Goebel, R.G. Sanfelice, and A.R. Teel. Hybrid Dynamical Systems: Modeling, Stability and Robustness. Princeton University Press, New Jersey, USA, 2012.

[16] J.P. Hespanha, P. Naghshtabrizi, and Y. Xu. A survey of recent results in networked control systems. In Proceedings of the IEEE, volume 95, pages 138-162, 2007.

[17] L.-S. Hu, J. Lam, Y.-Y. Cao, and H.-H.Shao. A LMI approach to robust $H_{2}$ sampled-data control for linear uncertain systems. IEEE Transactions on Systems, Man and Cybernetics, Part B: Cybernetics, 33(1):149-155, February 2003.

[18] I. Karafyllis and C. Kravaris. From continuous-time design to sampled-data design of observers. IEEE Transactions on Automatic Control, 54(9):2169-2174, 2009.

[19] V. Lakshmikantham, V. M. Matrosov, and S. Sivasundaram. Vector Lyapunov Functions and Stability Analysis of Nonlinear Systems. Mathematics and its Applications. Kluwer Academic Publishers Group, Dordrecht, 1991.

[20] J. Löfberg. Yalmip: A toolbox for modeling and optimization in MATLAB. In Proceedings of the CACSD Conference, Taipei, Taiwan, 2004.

[21] F. Mazenc, V. Andrieu, and M. Malisoff. Design of continuousdiscrete observers for time-varying nonlinear systems. Automatica, 57:135-144, 2015.

[22] F. Mazenc and T. Dinh. Construction of interval observers for continuoustime systems with discrete measurements. Automatica, 50:2555-2560, 2014.

[23] P. Naghshtabrizi, J.P. Hespanha, and A.R. Teel. Exponential stability of impulsive systems with application to uncertain sampled-data systems. Systems and Control Letters, 57(5):378-385, 2008.

[24] D. Nešić, A.R. Teel, and L. Zaccarian. Stability and performance of siso control systems with first-order reset elements. IEEE Transactions on Automatic Control, 56(11):2567-2582, 2011.

[25] A. Poznyak. Advanced Mathematical Tools for Control Engineers: Volume 1: Deterministic Techniques. Elsevier Science, Amsterdam-Boston, 2008. 
[26] H. Ríos, L. Hetel, and D. Efimov. Vector Lyapunov function based stability for a class of impulsive systems. In Proceedings of the 54th IEEE Conference on Decision and Control, pages 2247-2251, Osaka, Japan, 2015.

[27] H. Ríos, L. Hetel, and D. Efimov. Nonlinear impulsive systems: 2d stability analysis approach. Automatica, 80:32-40, 2017.

[28] E. Rogers, K. Galkowski, and D. Owens. Control Systems Theory and Applications for Linear Repetitive Processes, volume 349 of Lecture Notes in Control and Information Sciences. Springer-Verlag, Berlin, 2007.

[29] N. Sivashankar and P.P. Khargonekar. Characterization of the $\mathcal{L}_{2}$-induced norm for linear systems with jumps with applications to sampled-data systems. SIAM Journal on Control and Optimization, 32(4):1128-1150, 1994.

[30] J.F. Sturm. Using SEDUMI 1.02, a MATLAB toolbox for optimization over symmetric cones. Optimization Methods and Software, 11(12):625-653, 2001.

[31] N. Yeganefar, N. Yeganefar, M. Ghamgui, and E. Moulay. Lyapunov theory for 2-d nonlinear roesser models: Application to asymptotic and exponential stability. IEEE Transactions on Automatic Control, 58(5):12991304, 2013. 